\title{
Single cell analysis reveals altered tumor microenvironments of relapse- and remission- associated pediatric acute myeloid leukemia
}

\section{Gulay Ulukaya}

Aflac Cancer and Blood Disorders Center, Children's Healthcare of Atlanta, Atlanta, GA

\section{Beena Thomas}

Emory University

Swati Bhasin

Emory University

Hope Mumme

Emory University https://orcid.org/0000-0002-4171-1736

\section{Bhakti Dwivedi}

Emory University

\section{Pruthvi Perumalla}

Emory University

\section{Debasree Sarkar}

Emory University

Himalee Sabnis

Emory University

\section{Sunita Park}

Children's Healthcare of Atlanta

\section{Deborah DeRyckere}

Emory University SOM

\section{Sunil RaiKar}

Enory University

\section{Melinda Pauly}

Children's Healthcare of Atlanta

\section{Ryan Summers}

Children's Healthcare of Atlanta https://orcid.org/0000-0002-3621-8664

\section{Sharon Castellino}

Emory University

\section{Daniel Wechsler}

Emory University

Christopher Porter 
Department of Pediatrics, Emory School of Medicine

Douglas Graham

Emory University

Manoj Bhasin ( $\square$ manoj.bhasin@emory.edu )

Emory University

\section{Article}

Keywords: pediatric acute myeloid leukemia, relapse, remission, tumor microenvironments

Posted Date: September 3rd, 2021

DOl: https://doi.org/10.21203/rs.3.rs-819846/v2

License: (c) (7) This work is licensed under a Creative Commons Attribution 4.0 International License. Read Full License 
Single cell analysis reveals altered tumor microenvironments of relapse- and remissionassociated pediatric acute myeloid leukemia

Gulay B. Ulukaya ${ }^{1,2^{*}}$, Beena E. Thomas ${ }^{1,2^{\star}}$, Swati S. Bhasin ${ }^{1,2,3}$, Hope Mumme ${ }^{1,2}$, Bhakti Dwivedi ${ }^{4}$, Pruthvi Perumalla ${ }^{3}$, Debasree Sarkar ${ }^{1,3}$, Himalee S. Sabnis ${ }^{1,2}$, Sunita I. Park ${ }^{5}$, Deborah DeRyckere ${ }^{1,2}$, Sunil Raikar ${ }^{1,2}$, Melinda Pauly ${ }^{1,2}$, Ryan J. Summers ${ }^{1,2}$, Sharon M. Castellino ${ }^{1,2}$, Daniel S. Wechsler ${ }^{1,2}$, Christopher C. Porter ${ }^{1,2}$, Douglas K. Graham ${ }^{1,2}$, Manoj Bhasin\# $\#^{1,2,3,4,6}$

1. Aflac Cancer and Blood Disorders Center, Children's Healthcare of Atlanta, Atlanta, GA

2. Department of Pediatrics, Emory University, Atlanta, GA

3. Department of Biomedical Informatics, Emory University, Atlanta, GA

4. Winship Cancer Institute, Emory University, Atlanta, GA

5. Children's Healthcare of Atlanta, Atlanta, GA

6. Department of Biomedical Engineering, Georgia Institute of Technology, Atlanta, GA

${ }^{*}$ Equal Contribution

\section{\#Corresponding author:}

Manoj K. Bhasin, MS, PhD.

Aflac Cancer and Blood Disorders Center

Children Healthcare of Atlanta

Woodruff Memorial Research Building, Room 4107

101 Woodruff Circle, 4th Floor East

Emory School of Medicine

Atlanta, GA 30322.

Emails: manoj.bhasin@emory.edu

Telephone: (404) 712-9849

One Sentence Summary: Distinctive single cell profiles of heterogenous blast and immune cells associated with continuous complete remission and relapse in pediatric AML patients 


\begin{abstract}
:
Relapse- and continuous complete remission (CCR)-associated pediatric acute myeloid leukemia $(A M L)$ patient bone marrows collected at the time of diagnosis (Dx), end of induction (EOI) and relapse were analyzed by single cell RNA sequencing. A novel AML blasts-associated 7-genes signature (CLEC11A, PRAME, AZU1, NREP, ARMH1, C1QBP, TRH) displayed a strong correlation with blast percentages and overall survival in the TARGET AML dataset $(H R=2.3$; $P$ value=.007). Distinct clusters of AML-blasts at Dx were observed for relapse- and CCRassociated samples with differential expression of genes associated with survival. Relapseassociated samples demonstrated enrichment of exhausted T cells and M2 macrophages as opposed to inflammatory M1 macrophages in CCR-associated samples at Dx. EOI treatment resistant blast cells overexpressed fatty acid oxidation, tumor growth and stemness genes. Also, a relapse-associated EOI samples T cells subset showed downregulation of MHC Class I and regulatory genes. Altogether, this study deeply characterizes pediatric AML relapse-/CCRassociated tumor microenvironment transcriptome landscape.
\end{abstract}




\section{Introduction}

Acute myeloid leukemia (AML) accounts for about $20 \%$ of childhood leukemia(1). Improvements in outcomes for children with AML, attributed to intensification of frontline therapy, hematopoietic stem cell transplant (HSCT), and better supportive care, have not kept pace with those for children with acute lymphoblastic leukemia (ALL). The prognosis and treatment of pediatric AML remain challenging because of disease heterogeneity, high relapse rates, and therapy associated toxicity (2). It is important to identify novel risk stratification strategies that can be used to guide better therapy and improve outcomes for pediatric AML patients.

AML relapse can result from minimal residual disease (MRD) not detected/recognized by current diagnostic techniques including bulk gene expression analysis and imaging. The Therapeutically Applicable Research to Generate Effective Treatments (TARGET) AML initiative and large-scale genetic profiling studies have revealed that $A M L$ is a molecularly diverse disease having various gene mutations, insertions, and fusions associated with poor prognosis(3). Molecular characterization of the tumor microenvironment (TME) along with identification of biomarkers/targets that distinguish AML blasts cells (AML blasts) from non-malignant cells (nonAML cells) are critical for defining relapse-associated cell clusters and/or changes in the transcriptional landscape. The cellular composition of the TME and the transcriptional states thereof may contribute to and be affected by the leukemic process at the time of diagnosis (Dx) and the presence of residual blast cells at the end of induction (EOI) chemotherapy. Therefore, comparative analysis between Dx and EOI samples as well as a separate analysis of Dx and EOI samples is critical for characterizing the role of TME determinants in AML relapse/continuous complete remission (CCR).

We evaluated TME using single cell RNA sequencing (scRNA-seq) to identify relapse- and CCRassociated cells, gene alterations, and pathways at Dx, EOI, and relapse (Rel). Integrated scRNASeq analysis of paired Dx and EOI samples identified a 7-gene signature that distinguished AML blasts from non-transformed cells. Dx associated AML blasts demonstrated specific transcriptomic differences associated with relapse or CCR. Furthermore, distinct baseline characteristics of innate and adaptive immune cells at the time of diagnosis were associated with either CCR (monocyte/macrophage enrichment) or relapse (exhausted T cell enrichment). This study identified cell types associated with AML relapse/CCR and incidental novel genes and pathways, thus opens up avenues for the development of novel diagnostic and therapeutic targets in pediatric AML. 


\section{Results}

\section{Clinical samples and study design}

Frozen samples obtained from the Children's Healthcare of Atlanta Pediatric Biorepository were viably thawed and processed using 10X genomics platform for single cell profiling. The cohort of samples was made up of genetically and morphologically diverse cases of AML (Table S1A). A total of 23 bone marrow (BM) samples were processed from 15 patients (nine with CCR and six with relapse). Of the $23 \mathrm{BM}$ samples, 14 were collected at Dx, five were collected at EOI, and four were collected at relapse (Figure S1, Table S1B).

\section{Single cell analysis identified heterogeneous patient specific AML blasts}

ScRNA-seq analysis of four paired Dx and EOI samples (three from patients with relapse and one from a patient with CCR) was carried out. After preprocessing, alignment, and quality control steps including removal of low-quality reads and cells, the remaining 19,350 cells were analyzed using unsupervised and supervised approaches. The unsupervised analysis on preprocessed and normalized scRNA-seq data using Uniform Manifold Approximation and Projection (UMAP) approach (4) identified 14 single cell clusters (Fig. 1A). Ten of these clusters were manually annotated based on the expression of well-established gene markers as differentiated immune and stromal cells (Fig. 1B). The remaining clusters enriched with undifferentiated cells were putatively considered as blast cell clusters and annotated based on top cluster specific overexpressed genes (Fig. 1B, Figure S2). The differentiated immune cells included $\mathrm{T}$ cells $\left(C D 3 D^{+}, I L 32^{+}\right)$, B cells $\left(C D 19^{+}, C D 79 A^{+}, M S 4 A 1^{+}\right)$, monocytes (CD14 ${ }^{+}$; mono), macrophages (CD68 ${ }^{+}$; macro), monocytes/macrophages (CD14,$C D 68^{+}$; Mono/mac), plasmacytoid dendritic cells $\left(G Z M B^{+}, I L 3 R A^{+}, I R F 8^{+} ; \mathrm{pDC}\right)$, and neutrophils (CD63+) (Fig. 1B). The four undifferentiated blast cells exhibited overexpression of canonical blasts associated marker genes $\left(\mathrm{MPO}^{+}, \mathrm{CD}^{2} 3^{+}\right.$, $\left.C D 34^{+}\right)$and/or multiple lineage genes $\left(C D 3^{+}, C D 19^{+}, E L A N E^{+}\right.$, etc.) (Fig. 1B, Figure S2). Three of the four blast cell clusters, i.e., $\mathrm{MPO}^{+}, \mathrm{AZU}^{+}$, and $\mathrm{CD} 38^{+}$blasts, were sample specific as they were enriched with cells from individual patients (Fig. 1C) indicating significant heterogeneity among the blast cells. Additionally, the split UMAP analysis showed that these three canonical blast cell clusters were over-represented in the Dx samples compared to EOI samples (Fig. 1D). Also, these three Dx enriched blast cell clusters depicted high expression of well-established AML blast cells associated genes, namely, MPO and CD34 (Fig. 1E). In summary, scRNA-Seq data distinguished heterogeneous AML blast cells as well as immune cells in the Dx TME samples.

\section{Identification of AML blasts progenitor signature}

The supervised comparison of Dx enriched AML blast cell clusters and EOI enriched non-blast cell clusters (Fig 1D), based on the Wilcoxon rank test, identified a set of 232 significantly differentially expressed genes (DEGs) (adjusted $P$-value $<0.01$ and log $F C>1.2$ ). To further refine the gene list, we performed external validation analysis using the TARGET AML bulk transcriptome data (3) that contains Dx BM samples with different proportions of blast cells and EOI BM samples. The TARGET AML Dx samples were partitioned into 3 bins based on the extent of disease burden $(>60 \%, 30-60 \%,<30 \%$ blast cells) to determine the expression patterns of the identified 232 blast-related genes. 44 genes were significantly overexpressed in high blasts samples (>60\%) compared to EOI samples as well as high blasts samples compared to low blast $(<30 \%$ ) samples (FC >1.75 and P-value <0.05) (Figure S3, Table S2). We further evaluated the expression patterns of these 44 genes in the blast cell clusters in scRNA-Seq data (Fig 1F, Table S3). Of the 44 genes, 20 genes depicted pattern of specific overexpression in the blast cell 
clusters suggesting the existence of a common progenitor AML blast cell signature cross heterogeneous blast cells (Table S4). A heatmap depicting the expression pattern for these 20 blast specific genes in TARGET AML data is shown in Fig. 2A. The blast cells overexpressed genes include genes related to anti-apoptosis (PRAME, MSLN, CITED4), growth factor for progenitor hematopoietic cells (CLEC11A), cell proliferation (CAPRIN1), and PPARa-induced proliferation and tumor growth (FABP5) (Fig. 2A). Feature-plot of two of the identified blast associated genes (NREP and CLEC11) depict uniform expression in the heterogenous blast clusters (Fig. 2B) that is equivalent to or better than some of the genes used clinically for identifying AML blasts (CD34, MPO, CD33, and CD56/NCAM1) (Fig. 1E, Figure S4). The blast associated genes expression exhibited correlation with the blasts percentages in the AML BM samples (Fig. 2C). Also, majority of the blast associated genes exhibited significant association with overall survival (OS) in the TARGET AML data set (Table S5, Fig. 2D) indicating their possible involvement in AML progression.

To verify blast cells characterization using our 20-gene signature, we also looked at blast cell clusters in longitudinal samples (Dx, EOI, Rel) from two patients with negative MRD at EOI. The analysis revealed significantly fewer blast cells in the EOI samples as compared to Dx and Rel samples; the split UMAP (Fig. 2E) and bar plot (Fig. 2F) shows the two immature blast cell clusters (MPO+ and $A Z U 1+)$ that predominate in Dx and Rel samples are minimally represented in EOI samples. To generate a more concise AML blast specific signature and evaluate performance in discriminating AML blast cells from other cells, we implemented supervised machine learning approach, support vector machine (SVM), and calculated its performance using cross-validation. SVM is a powerful supervised machine learning technique for identifying multigene biomarker panels from complex datasets $(5,6)$. The analysis identified a set of 7 genes (CLEC11A, PRAME, AZU1, NREP, ARMH1, C1QBP, TRH) that discriminated AML associated blast cells from non-AML cells with an AUC of 0.968 (Fig. 2G, Table S6). Several of these genes, including CLEC11A (7), PRAME (8), and AZU1 (9) have been previously associated with AML. The analysis also identified a unique gene, ARMH1/C1orf228, that codes for a yet uncharacterized protein and has not been previously reported to be associated with pediatric or adult AML. NREP is also known to affect cell development (10), while PRAME may alter metabolic and immune pathways (11). This unique set of genes overexpressed in AML blasts are therefore associated with a myriad of cell functions that promote tumor growth and spread. The module score based on 7-gene signatures effectively distinguished AML blast cells from non-AML cells as only $\mathrm{MPO}^{+}$and $\mathrm{AZU}^{+}$blasts clusters had positive module scores (Figure S5). The clinical significance of this 7-gene signature was tested by evaluating correlation with survival in the TARGET AML data. Patients having high expression of the selected 7 genes had a significant correlation with poor survival $(\mathrm{HR}=2.3$ and Log Rank P-value=.007) compared to patients with low expression of these genes (Fig. 2H, Table S7A).

\section{Comparative analysis of Dx relapse- and CCR-associated samples}

We performed a comparative analysis of 14 bone marrow samples collected at Dx, of which 6 later relapsed (1D - 6D) and 8 remained in CCR (7D - 14D) (Figure S1, Table S1). After quality control, processing, and normalization, 28,181 cells clustered into 15 distinct clusters (Figure S6A). Based on the 7-gene blast signature module score, 14,166 cells were classified as AML blast cells and the remaining 14,015 cells were classified as non-AML cell clusters (Figure S6B). The UMAP projection depicted that CCR-associated clusters 1,3,4,6,8,10,14 and relapse- 
associated clusters $0,2,5,7,9,11,12,13$ clustered separately, suggesting differential transcriptome makeup of the 2 sets of blast cells (Fig. 3A). Also, we observed patient specific clusters indicative of inter-patient variation in the blast cells profiles (Fig. 3B). Differential expression analysis of select relapse-associated samples enriched clusters $(0,2,7,13)$ and CCR-associated samples enriched clusters $(1,3,4)$ revealed significant transcriptome differences (Fig. 3C). The CCRassociated clusters exhibited high expression of genes including MPO, IFITM3, TRH, PRTN3, HLA-DPM1, and NPM1 while relapse-associated clusters expressed high levels of genes including CRIP1, FLNA, and RFLNB/FAM101B (Fig. 3C). Survival analysis using the SurvivalGeneie tool TARGET AML dataset indicated that genes like FLNA (cytoskeleton component, signaling scaffold and Pol1 transcription regulator) and RFLNB/FAM101B (formation of cartilaginous skeletal elements) overexpressed in relapse-associated cell clusters were significantly associated with poor OS (Fig. 3D, supplementary table 5C). On the other hand, genes overexpressed in the CCR-associated clusters such as MPO (microbicidal activity of neutrophils) and $T R H$ (controls the secretion of thyroid-stimulating hormone) were significantly associated with better OS (Log Rank $P$ value $=0.001,0.000396$ for MPO, TRH respectively, Table S7B, Fig. 3D). Additionally, higher expression of RFLNB/FAM101B genes showed a significant correlation with shorter EFS while lower expression was associated with intermediate EFS (Figure S7A). The interactive string network analysis based on known and predicted protein-protein interactions (13) identified FLNA as a key interactant of RFLNB/FAM101B. Another identified interactant of RFLNB/FAM101B was WDFY4, which plays a critical role in the regulation of classical dendritic cells (CDC1) mediated cross-presentation of viral and tumor antigens (14) (Figure S7B). The Kaplan-Meier plots showed a significant correlation of high expression of combined RFLNB/FAM101B and WDFY4 genes with poor OS (Log Rank P-value=0.017) and shorter EFS (Log Rank P-value=0.00094) (Figure S7C, D; Table S7A). Furthermore, pathways analysis on relapse-associated blast cells differentially expressed genes depicted significant activation (P-value <0.01) of multiple pathways including "RhoGDI signaling", "eNOS signaling", "Androgen Signaling", and "Protein Kinase A signaling" (Fig. 3E). These relapse-associated blast cells also depicted significant inhibition of pathways related to JAK/STAT signaling, HIF1- $\alpha$, Interferon, and neuroinflammation signaling (Fig. 3E). Further upstream regulator-based systems biology analysis identified activation of a highly connected cohesive network of cell growth and proliferation related master regulators (e.g., MTA1, NKX2.3, TCF3) in relapse-associated blasts (Fig. 3F). The analysis also depicted significant inhibition of multiple immune and metabolism related key molecules including HIF1A, IRF7, STAT1 (Figure S7E) in relapse-associated samples enriched AML blast cells.

\section{Dx relapse-associated non-AML clusters depicted enrichment of exhausted $\mathrm{T}$ cells and diminution of M1 macrophages}

Next, we performed focused analysis on the TME non-AML cells in the 14 Dx samples (Fig. 4A). Clusters were annotated based on the expression of specific gene markers (Fig. 4B). CCR-associated clusters had a higher representation of monocyte/macrophages $\left(C D 14^{+}, C D 16^{+}\right)$ while relapse-associated clusters depicted a higher proportion of T cells $\left(C D 3^{+}\right)$(Fig. $\left.4 \mathrm{C}\right)$. These results suggest differences in the type of immune cells populating the TME at the time of Dx in those who go on to achieve CCR as compared to those who eventually suffered relapse.

To understand if a particular subtype of T cells is associated with AML relapse/CCR, we sub-clustered Dx T cells (cluster 0, Fig. 4A) using Monocle package (15) which revealed 11 distinct subclusters of $\mathrm{T}$ cells (Fig. 5A). Most of the patients showed separate subclusters for 
naïve and activated T cells (Fig. 5B, Figure S8A). While naïve T cell $\left(C C R 7^{+}, L E F 1^{+}, T C F 7^{+}\right)$subclusters 2, 4 and 7 are $C D 8^{+}$, sub-clusters 1 and 3 were $C D 4^{+}$clusters (Figure S8A, B). Clusters $5,6,9,10$, and 11 were activated T cell clusters expressing CCL5, KLRB1, KLRD1, GZMH, CD69, and CD44 (Fig. 5B). The unequal distribution of CCR- and relapse- associated samples in different clusters in the T cell subset is suggestive of relapse- and CCR-associated transcriptome variations in T cell sub-populations (Fig. 5B). Comparative analysis between relapse- and CCRassociated samples revealed that the former had a significantly higher percentage of $\mathrm{T}$ cells (Fig. 5C). Also, $T$ cell exhaustion estimation based on ssGSEA score $(16,17)$ revealed significantly higher exhaustion of T cells $(P$ value $<0.0001)$ in relapse-associated samples compared to CCRassociated samples (Fig. 5D). The relapse enriched clusters (sub-clusters 5 and 6 ) depicted overexpression of $\mathrm{T}$ cell exhaustion marker genes including HAVCR2, LAG3, PDCD1, NFATC1, TIGIT, and TOX (Figure S8B). The relapse-associated samples dominant clusters 1 and 11 depicted overexpression of $C D 69$, a type II glycoprotein that is known to regulate inflammation and exhaustion of tissue resident T cells and promoting tumor growth/relapse (18). On the other hand, significantly more naïve T cells (CCR7, LEF1, TCF7) were observed in the CCR-associated T cell subsets (Fig 5D). Further GSEA $(16,17)$ analysis depicted higher proportion of cells with $T$ regulatory signature in the patients with relapse as compared to patients with $C C R$, hinting toward immunosuppression in patients that eventually had a relapse (Fig 5E). Comparative analysis of relapse-associated sub-clusters 3,5, and 6 and CCR-associated sub-clusters 7,8, and 10 identified significant differentially expressed genes (DEGs) (Fig 5F). Relapse-associated subclusters showed enhanced expression of genes associated with effector T cells (CCL5, NKG7, GNLY, GZMA, GZMK, NFATC1), ubiquitin gene; UBC associated with cell differentiation and certain immune cell functions (19), inflammation associated and mitogen inducible gene, DUSP2 (20). On the other hand, CCR-associated clusters showed higher expression of naïve $T$ cell marker genes (LEF1, TCF7, CCR7, SELL), microtubule associated gene; RPL41 (21), scaffold protein gene; $A B L I M 1$ (Fig. 5F), The pathway analysis of relapse-associated upregulated DEGs revealed upregulation of multiple immune regulatory pathways including Th1 pathway, calcium induced T lymphocyte apoptosis, and to a lesser extent, upregulation of T cell exhaustion pathway with downregulation of PD-1 and PD-L1 cancer immunotherapy pathways (Fig. 5G). These results suggest that $T$ cells at Dx exhibit relapse- and CCR-associated specific enrichment of $T$ cell subtypes that may play a role in the outcome in AML.

\section{Inflammatory monocytes/macrophages enriched in CCR-associated samples}

Focused analysis of Dx non-AML cells identified three monocyte/macrophage cell clusters with differential enrichment of samples from those with CCR compared to those with relapse (Fig. 6A). Clusters 1 and 2 were enriched with CCR-associated Dx samples while cluster 7 was almost exclusively comprised of relapse-associated Dx samples (Fig. 6A, Figure S9A). Cells in cluster 7 overexpressed many of the 20-gene signature genes including PRAME, CITED4, CLEC11A, $K C N E 5$, and MFSD10, unlike clusters 1 and 2, indicating the immaturity of these cells (Figure S9B). Cluster 7 also demonstrated expression of multiple immature marker genes including MEIS1, MSI1, PROM1, and EGFL7 (Figure S9C). Further DEGs analysis between CCR- and relapse-associated samples enriched monocytes/macrophages revealed upregulation of S100A inflammatory genes family and protease inhibitors, TIMP1 and CST3 in the CCR-associated samples enriched clusters (Fig. 6B). The analysis for canonical M1 and M2 macrophage markers revealed that CCR-associated clusters depicted overexpression of $\mathrm{M} 1$ macrophage markers (IL6, CD86, TNF, IL1B, FCGR2A, CSTA, S100A8, S100A9, S100A12, TYROBP, FCN1), while the relapse-associated cell cluster 7 mostly depicted overexpression of M2 macrophage markers 
(MRC1, ARG1, MMP9) (Fig. 6C). Further upstream regulator analysis of DEGs from the CCRassociated samples enriched clusters revealed activation of multiple M1 macrophage polarization related key regulators including FOS, TREM1, CD44, and IL1B along with activation of the inflammatory response associated $N F \kappa \beta 2$ (Fig. 6D). The analysis also depicted the downregulation of key regulators related to $M 2$ macrophage suppression including SAMHD1, HOXA9, and SATB1 (Fig 6D) CCR-associated samples enriched clusters. SAMHD1 has been shown to play a critical role in inhibiting innate immunity through inhibition of NF-k $\beta$ and interferon pathways (22). Further evaluation of gene expression levels for key regulators FOS, TREM1, and $N F K B I$ depicted high expression in CCR-associated samples while CAT and SAMHD1 were higher in the relapse-associated samples (Fig. 6E). To summarize, Dx CCR-associated samples are enriched with inflammatory M1 macrophages, whereas relapse-associated samples depicted enrichment of immature monocyte population of cells exhibiting mixed M1/M2 lineage markers.

\section{Characterization of treatment-resistant residual cell population at EOI}

Sc-RNA-Seq analysis of EOI samples from patients with relapse $(n=3)$ and CCR $(n=2)$ generated 15,070 single cell profiles. The analysis identified 13 distinct cell clusters based on trancriptome profile (Fig. 7A) that were annotated based on enrichment of 20 blast overexpressed genes (Figure S10A) and expression of canonical cell specific marker genes (Fig. 7B). The module score-based classification using the 7-gene blast signature identified enrichment of blast cells in four clusters with a cut-off value of scaled ssGSEA score set at $>0.25$ (Fig. 7C). To identify residual blast cells, we performed an integrated analysis of single cell data obtained at the time of $\mathrm{Dx}$ and EOI. The analysis assisted in identifying blast cells present before (Dx) and after treatment (EOI), namely clusters 4,6 , and 8 (Figure S10B). Analysis showed persistence of few treatment-resistant blast cells ( $\sim \%$ of the blast cells present in Dx) in EOI samples (Figure S10B). These AML blasts overexpressed multiple AML blast genes validating AML blast characteristics for these cells (Figure S10C).

To further determine the transcriptome landscape of treatment-resistant residual tumor cells, we performed comparative gene analysis of treatment-responsive (only detected in Dx samples) and treatment-resistant (detected in both Dx and EOl samples) cells (Fig. 7D). The treatment resistant blast cells depicted overexpression of multiple genes associated with tumor growth and poor outcome including HOPX, SELENOP/SEPP1, and FAM3OA/C1orf110. Survival analysis on these treatment-resistant residual tumor cells overexpressed genes in the TARGET AML dataset showed significant association with poor OS as well as lower EFS (Figure S11, Table S8). Additionally, multiple genes (MPO, KCNE5, MSLN, and TRH) that are significantly upregulated in treatment responsive blast cells are associated with better OS in AML (Fig. 3D, 7D, Figure S11). Further pathways analysis of the DEGs depicted significant upregulation of pathways related to muscle contraction, fatty acid omega oxidation, PPARa network in the treatment resistant cells (Fig. 7E, Table S9). Survival analysis based on treatment resistant cells upregulated pathways (e.g., fatty acid omega oxidation and PPARa network) in the TARGET AML dataset showed significant association with poor survival indicating their role in disease progression (Table S10). Additionally, upstream regulator analysis on DEGs in treatment-resistant cells depicted activation of multiple key regulators associated with epithelial-mesenchymal transitions including SOX4, STAT3, and TGFß1 (Fig. 7F).

To summarize, treatment-resistant blasts identified in this preliminary analysis may contribute to relapse by differential regulation of genes and pathways associated with modulation of TME, leading to poor OS and lower EFS in AML patients. 


\section{Relapse-associated EOI samples depicted enrichment of T cells and diminution of monocytes/macrophages}

EOI samples showed a much larger proportion of non-AML immune cells since therapeutic intervention (chemotherapy) results in the killing of tumor cells. To further characterize EOI single cell landscape, the focused analysis on non-AML cells was performed after removing residual blast cell clusters discussed in the previous section. The split UMAP of CCR- and relapseassociated samples revealed the altered distribution of the transcriptionally distinct canonical immune cells (T cells, NK cells, and macrophages/monocytes) (Fig. 8A). Analysis of relapse- and CCR-associated non-AML clusters at EOI revealed a similar pattern to that seen at Dx, that is higher enrichment of T cells in relapse-associated samples and a higher proportion of monocytes and macrophages in the CCR-associated samples enriched clusters (Fig. 8B, C). Further M1 and M2 macrophage enrichment analysis revealed a highly significant $(p<0.001)$ inflammatory M1 macrophages predominance in CCR-associated EOI samples and significant $(p<0.05)$ M2 macrophages enrichment in relapse-associated EOI samples (Fig. 8D). The T cells formed three distinct clusters i.e., naïve T cell-1, naïve T cell-2, and NKT cells clusters (Fig. 8A, Figure S12A). Naïve $T$ cells-2 cluster depicted significantly $(P$ value $<0.05)$ higher enrichment in the relapseassociated EOI samples (Figure S12B). The comparative analysis of the two naïve T cell clusters depicted that relapse-enriched naïve T cell-2 has lower expression of $\mathrm{MHC}$ class I genes including HLA-A and HLA-B (Figure S12C). Pathway analysis of naïve T cell-2 depicted downregulation of pathways related to Th1, Th2, calcium-induced T cell apoptosis, CD28 signaling in T helper cells, and integrin pathways (Fig. 8E).

\section{Discussion}

ScRNA-Seq has revolutionized cancer research and is especially pertinent for AML research due to the tumor and microenvironment heterogeneity. We analyzed a heterogeneous group of AML samples; AML with identified mutations, AML associated with myelodysplasia, AML showing minimal differentiation, and AML NOS (Table S1). Our analysis indicated that AML blast cells are significantly heterogeneous and patient specific (Fig 3A). Despite the heterogeneity, supervised analysis on blast cells using a machine learning approach identified a unique 7-gene blast progenitor signature that is overexpressed in the heterogeneous AML blast populations studied. The expression of signature genes depicted a significant correlation with the percentage of blast cells in external TARGET AML datasets demonstrating specific expression in AML blast cells. The 7-gene signature included genes coding for growth factors (CLEC11A), myeloid differentiation factor (AZU1), cell development (NREP), and transcription factors (PRAME). TRH has been shown to have high expression in AML (24) while the function associated with $\mathrm{ARMH} 1 / \mathrm{C} 1$ orf228 is not known yet. The functional variability of the 7-gene signature capable of distinguishing AML blasts from non-AML cells is suggestive of multiple synergistic pathways being modulated in these AML blast cells. Significant association of the 7-gene signature overexpression with poor OS can be attributed to the regulation of a myriad of cell functions that promote tumor growth, proliferation, and migration. Analysis of longitudinal samples (Dx, EOI, and Rel) showed that blast cells identified at Dx reduced significantly at EOI and reappear in Rel samples. The blast cells in the Rel samples display significant similarity with Dx blast cells indicating that expansion of certain therapy resistant rare Dx blast cells clones that might be responsible for disease relapse.

ScRNA-Seq data analysis revealed distinct gene expression profiles at Dx of blasts from patients that are destined to relapse vs. remain in CCR. The blast cells of relapse-associated samples 
even at the time of disease diagnosis depicted upregulation of multiple genes related to cytoskeleton formation (FLNA, RFLNB/FAM101B) associated with poor clinical outcome (Table S7B). Cytoskeleton proteins affect various tumor cell characteristics like cell survival because of drug resistance, invasiveness, and metastasis (25). Also, actin cytoskeleton remodeling can change physical and functional interactions at the immunological synapse (26). Microtubule targeting or disrupting agents have been tested for a variety of therapy resistant cancers (27). One such drug, Combretastatin A4-phosphate (CA4P) targets VCAM1, vascular cell adhesion molecule 1, expression and resulted in AML cell line apoptosis (27). RFLNB belongs to the refilin family, short lived F-actin regulators, that control cell fate specification and differentiation (28). RFLNB promoter binds transcription factor PKNOX1/PREP1 that regulates embryonic development and determines cell fate (29). RFLNB and PKNOX1 are also known to modulate responsiveness to TGF $\beta$ and promote epithelial mesenchymal transition (30). RFLNB targets FLNA to organize a perinuclear network and stabilize nuclear shape (31). FLNA is known to interact with integrin, ITGB7 that interacts with VCAM1 (32). Therefore, overexpression of FLNA, RFLNB in blasts cells may modulate the cell structure and subsequent interactions with immune cells. These changes influence cell junction organization, cell motility and activate nuclear transcription programs that may contribute to AML relapse. The genes overexpressed in the relapse-associated blasts cells were found to correlate with upregulation of multiple pathways including RhoGDI, eNOS, and protein kinase A signaling. Rho GDP-dissociation inhibitor (RhoGDI) pathway activation has been shown to enhance progression chemoresistant cancer cell lines and animal models (33). Studies also show that endothelial NOS (eNOS) can affect tumor processes such as resistance, angiogenesis, invasion, and metastasis. An increase in eNOS expression is found to have associations with poor relapse-free and overall survival as well (34). In contrast, Dx blasts from samples with CCR showed significant upregulation of genes like MPO and TRH that are associated with better survival outcomes. Previously TRH has been shown to be upregulated in $\mathrm{t}(8 ; 21)$ AML subtype and high expression is associated with better clinical outcomes (35). TRH has been implicated to have a role in the immune system (36), but its role in AML blasts is not clear. MPO is the key enzyme of myeloid lineage commitment and associated with a favorable prognosis in AML (37). This differential expression of genes identified in relapse- and CCR-associated blasts and subsequent differential regulation of pathways and upstream key transcriptional regulators may contribute to differences in hematopoietic niches that result in AML relapse/CCR.

Additionally, investigation of paired Dx and EOI samples led to the identification and characterization of therapy resistant residual blast cells. The therapy resistant blast cells depicted overexpression of multiple genes associated with tumor growth (SELENOP), metabolism, and stemness (HOPX, FAM3OA). The higher expression of HOPX is associated with higher platelet counts, lower white blood cell counts, lower lactate dehydrogenase levels, lower complete remission rates, and poor overall survival (38). SELENOP encodes for a selenoprotein that mediates microenvironmental changes that influence tumor growth and prognosis across multiple solid tumors such as prostate and breast cancers (39). Similarly, the FAM30A gene has been recently shown to be associated with leukemia stemness and high-risk pediatric AML (40). The pathways analysis of treatment resistant blast cells showed upregulation of multiple pathways including fatty acid oxidation, and PPARa network. Fatty acid oxidation has been linked to enrichment and survival of leukemic stem cells resulting in disease progression and relapse. Furthermore, the resistance of AML to conventional and metabolically oriented therapies (i.e., Venetoclax) has also shown an association with upregulation of fatty acid oxidation in the 
leukemic stem cells (LSCs) $(41,42)$. Therefore our preliminary data support targeting fatty acid metabolism in AML LSCs for achieving CCR though more validation is required.

AML is mainly characterized by enrichment of immature myeloblasts, but immune cells play an integral role in the disease progression and outcome (43). Mature myeloid lineage cells have been implicated in altering $\mathrm{T}$ cell phenotypes and generating a immunosuppressive microenvironment in $\operatorname{AML}(44,45)$. Additionally, dysregulation of crosstalk between tumor and stromal cells has been shown as key toward tumor proliferation or suppression. To explore the impact of TME at the baseline, we performed focused analysis on immune cells collected at Dx from patients with CCR and relapse outcomes. Overall, T cells were more abundant in relapse-associated samples while monocyte/ macrophages were enriched in samples with CCR (Fig. 4). CCR-associated samples depicted naïve T cells enrichment compared to relapse-associated samples, which may contribute to effective resolution of the blast cells leading to remission. Samples from those with relapse with enriched exhausted $T$ cells will have an immunosuppressive TME resulting in less effective blast cells clearance. Also, pathway analysis showed enhanced Th1 pathways and costimulatory ICOS/ICOS-L T helper pathway indicative of a more inflammatory T-cell pattern in samples from those with relapse. ICOSL expression in the AML cells has been shown to result in $\mathrm{ICOS}^{+}$Tregs expansion that promotes immune evasion as well as IL-10 secreted by ICOS ${ }^{+}$Tregs support AML cells proliferation (46). Further analysis of samples collected at EOI suggested a shared pattern in immune cells enrichment at EOI and Dx; there were more T cells in samples that relapse later and higher monocyte/macrophages in samples with sustained remission. The transcriptionally distinct naïve $T$ cell clusters enriched in samples from those with relapse ( $T$ cell2) may be influenced by the type of blasts present in these samples.

The analysis of innate immune cells depicted enrichment of inflammatory M1 macrophages in Dx and EOI samples with CCR compared to samples with relapse, indicates that initial activation of these adaptive immune cells may contribute to remission. Leukemia associated macrophages (LAM) have been associated with survival and drug resistance of AML cells (47). It has been shown that targeting IRF7/SAPK/JNK pathway can promote M1 characteristics in LAM resulting in prolonged survival of leukemic mice. Conversely, relapsed EOI samples had more tolerant M2 macrophages that may not clear up the remaining blast cells that drive disease relapse. These findings indicate a potential role of innate immune cells in achieving deep and sustained remission and possible exploration for therapeutic intervention.

\section{Conclusions}

Common transcriptome and pathways present in the highly heterogenous AML sub-types enabled the usage of novel 7-gene signature for identifying AML blast cells in the current study. At the same time, distinct differences were identified in AML associated blasts as well as non-AML cells that distinguished samples with relapse from samples with CCR. We uncovered the presence of patient specific blasts at Dx, treatment-resistant blasts at EOI, and the presence/activation of different arms of the immune system (monocyte/macrophage and T cells) in samples from those with relapse and those with CCR at both Dx and EOI. The identification of upregulation of novel genes like $A R M H 1$ in $A M L$ blasts is important for diagnostic and therapeutic purposes. Using ScRNA-Seq we were able to identify unique gene signatures, characterize AML associated blasts and delineate immune cells make-up in the TME of children with different AML outcomes. 


\section{Material and Methods}

\section{Clinical Samples}

Samples were obtained as part of a precision medicine study of Aflac Cancer and Blood disorder center that was approved by the Emory institutional review board. Bone marrow core biopsies were obtained at the initial presentation of $A M L(D x)$, at the time of therapy remission; the end of induction (EOI), and at the time of relapse (Rel) as part of routine hematopathology evaluation. The study was performed on 15 patients, out of which 9 patients remain in continuous clinical remission, and 6 patients relapsed. To study the sequential changes in single cell landscape, we paired samples from two patients collected at Dx, EOI, and Rel. The single cell analysis at Dx involved 14 patients: six with relapse and eight with CCR. For EOI the analysis was performed on five patients' samples: three with relapse and two with CCR.

\section{Single Cell RNA Sequencing and analysis (Details in Supplementary Methods)}

We performed scRNA-Seq on viably revived bone marrow samples using the 10X Chromium 3'V3 and 5' $\mathrm{V} 1$ reagent kits and sequencing was performed using the massively parallel sequencing on the Novaseq S4 platform. Raw scRNA-Seq data was demultiplexed, aligned to the human reference genome (hg38), and processed for single cell gene counting using the Cell Ranger Software from 10X Genomics Inc. The single cell count data was normalized using the SCTransform function in Seurat v3.0 Bioconductor package (48) that uses regularized negative binomial models for normalizing sparse single cell data. The normalized expression profiles of the samples were merged, and batch corrected (if required) based on shared embedding using harmony Bioconductor package (49). Further quality control, pre-processing, unsupervised and supervised analysis on the data was performed using various $\mathrm{R}$ and Bioconductor packages. In an unbiased approach, cell types and subtypes signatures were generated by comparing the expression profile of target cell type with the rest of the cells using the non-parametric Wilcoxon rank test $(P$-value $<.01)$ and fold change $>1.2$.

To determine the cell specific gene dysregulations that are associated with relapse and CCR, we performed comparative analysis of the transcriptome profile of each cell type between relapsed and CCR groups. The comparative analysis of normalized data was performed using the nonparametric Wilcoxon rank model, based on raw $\mathrm{P}$ values and absolute fold change. Genes were considered significantly differentially expressed if $P$ values were $<0.05$ and $F C>1.5$.

\section{Pathway and Systems Biology Analysis}

To precisely characterize the AML blast cells and understand the molecular mechanism of disease progression, we performed pathways enrichment and systems biology analysis (See details in supplementary methods).

\section{Gene Set Enrichment Analysis}


To determine the enrichment of pre-determined gene sets, such as 7-gene signature and canonical pathways from the molecular signature database(16), we performed single sample gene set enrichment analysis (SSGSEA)(16) using escape package from the Bioconductor library. SsGSEA enrichment score represents the degree to which the genes in a particular gene set are coordinately up- or down-regulated within a cell cluster, which could serve as an indicator of gene set activity. To interrogate the unique pathways/gene sets that are differentially enriched in one cell type vs. other, we performed statistical analysis using student t-test or LIMMA (51) to select significantly differentially enriched gene sets. Pathways/Gene sets with an adjusted P-value $<0.01$ were considered statistically significant.

\section{Validation of scRNA-Seq blast enriched genes on external pediatric AML dataset}

The preliminary comparative analysis of AML Dx specific clusters identified a long list of AML enriched genes that are overexpressed in AML blast cells. To reduce the false positives and identify robust $A M L$ blast associated genes, we evaluated their pattern on the external pediatric AML dataset from TARGET initiative. The study contains bulk RNA sequencing data generated from bone marrow samples of $>\mathbf{3 0 0}$ AML subjects that include infants (<3 years old), children (314 years old), and adolescents/ young adults (AYA; 15-39 years old). The raw TARGET dataset was normalized using VOOM algorithm (52). To explore the expression pattern of single cell profiling identified AML blast associated genes in TARGET AML dataset, we binned the Dx datasets into different groups - based on percentages of blast cells (>60\%, $60 \%-30 \%,<30 \% s$ ). We hypothesized that genes that are specifically expressed by blast cells will depict a progressive downregulation pattern from high to low blast and EOI samples. The significance of progressive downregulation from high to low blast/EOI groups was determined using unpaired Student t-test (P-value). Genes with fold change $>0.7$ (High blast vs EOI) and $P$ value $<0.05$ were considered significantly associated with blast cells. All analysis was performed using different Bioconductor Packages and R language including LIMMA, and gplots (53).

\section{Survival analysis of AML blast genes}

To determine the association of blast genes with clinical outcomes in AML, we performed the survival analysis on the TARGET-AML dataset. For gene-level analysis, we used the median $\left(50^{\text {th }}\right.$ percentile) cut-off or an optimal cut point (cutp) estimated based on martingale residuals (54) using the 'survMisc' package in Bioconductor/R to separate the patients into high and low gene expression groups. For combined analysis, we used the non-parametric method, single sample gene set enrichment analysis (ssGSEA) as implemented in the GSVA package (55) to obtain a gene-set enrichment score. Overall Survival (OS) or Event-Free Survival (EFS) statistical analysis was performed using the 'survival' R package (See details in supplementary methods).

\section{Generation of AML blast associated 7-gene signature using Support Vector Machine}

To identify the key genes associated with AML blast we developed classifiers using the support vector machine technique $(5,6)$. The classifiers assist in identifying the genes that are most important for identifying the pediatric AML blast cells. Each classifier was developed based on the selected set of 20 genes that are overexpressed in blast cells at single cell level as well as in the bulk TARGET dataset. Classifiers were trained using normalized and preprocessed single cell 
data. For example, classifier for discriminating between blast and non-blast cells was trained based on input matrix of 20 gene expression from AML blast and non-AML cells. The performance of these classifiers in the training dataset were evaluated using Out of bag (OOB) cross-validation. The performance of classifiers was measured using threshold-independent receiver operating characteristic $(\mathrm{ROC})$ analysis. The analysis assisted in identifying a minimal set of genes that can distinguish blast cells from other cells with high Area Under Curve (AUC) from ROC analysis. 
Figure Legends:

Fig. 1: Single cell transcriptional profiling identifies three heterogenous putative AML blast cell clusters by comparative profiling of paired Diagnostics (Dx) and End of Induction (EOI) bone marrow samples. A. UMAP embedding of the paired Dx and EOI samples consisting of 19,350 high quality single cells portioned into 14 clusters. These clusters are colored based on canonical cell types gene markers (Fig. 1B) that include T cell $\left(C D 3 D^{+}, I L_{2} 2^{+}\right), \mathrm{B}$ cells $\left(C D 19^{+}\right.$, $\left.C D 79 A^{+}, M S 4 A 1^{+}\right)$, monocyte $\left(C D 14^{+}\right)$, macrophages $\left(C D 68^{+}\right)$, macrophage/monocyte $\left(C D 14^{+}\right.$, $\left.C D 68^{+}\right)$, plasmacytoid dendritic cells-pDC $\left(G Z M B^{+}, I L 3 R A^{+}, I R F 8^{+}\right)$, and neutrophils $\left(C D 63^{+}\right)$. The blast cells were annotated based on expression of top overexpressed gene. B. Dot plot showing expression of different cell type specific marker genes that were used to annotate the cell types (Red: high, Yellow: Mid, Cyan: low). C. Relative proportion of cells of different samples in each cell type cluster. Most of the blast cell clusters are made up of cells from 1-2 patient samples indicating AML blast heterogeneity. On the other hand, most of the immune cell clusters contain cells from multiple patient samples. D. Split UMAP showing the putative undifferentiated blast cells that are significantly over-represented in Dx samples (Dx enriched) and reduced in the EOI samples. E. Expression of clinically validated blast cell markers (MPO and CD34) across single cell clusters. Clusters with positive expression of marker genes are lassoed. CD34 expression is highly heterogenous. F. Heatmap of selected set of 44 genes showing significant overexpression (Fold Change $>1.2$, Adjusted $P$ value <.01) in Dx Enriched blast cells in comparison of other differentiated cell types. Relative gene expression is shown in pseudo color, where green represents downregulation, and red represents upregulation.

Fig. 2: Development of blast progenitor signature from heterogenous AML blast cells. A. Heatmap of 20 genes that depicts significant overexpression in the TARGET AML samples with high blast enrichment $(>60 \%)$ as compared to low blast $(<30 \%)$ and EOI samples. B. Feature plot of selected genes (CLEC11A, NREP) from 20 gene signature showing uniform overexpression in the heterogenous blast clusters. C. Representative expression profiles and $\mathbf{D}$. survival correlations of select genes (CLEC11A, NREP) showing a progressive downregulation pattern from high blast enrichment to low blast and EOI TARGET AML samples. The significance of the enrichment was calculated using Wilcox Rank t-test ( ${ }^{*} p$-value $<0.05,{ }^{* *} p$-value $<0.01,{ }^{* * *}$ $\mathrm{p}$-value $<0.001)$. The higher expression of these CLEC11A $(\mathrm{HR}=1.9,95 \% \mathrm{Cl}: 1.1-3.4, P=0.03)$ and NREP (HR=1.9, 95\% Cl: 1.2-3.1, $P=0.007)$ is associated with poor survival. E. UMAP plot depicting the blast cells identification based on the newly identified 20-gene overexpressed in blast cells (lassoed) of the longitudinal samples (DX, EOI, REL) from two patients. F. Bar plot showing fraction of cell types in DX, EOI and Rel samples. G. Receiver operative Curve (ROC) depicting performance of Support Vector Machine (SVM) approach based 7 genes signature (CLEC11A, PRAME, AZU1, NREP, ARMH1, C1QBP, TRH) to discriminated AML blast cells from other cells. H. Survival analysis depicted that patient with higher gene set enrichment score based on identified 7 genes exhibited poorer overall survival $(\mathrm{HR}=2.3,95 \% \mathrm{Cl}=1.2-4.3, P=0.0073)$ in the TARGET AML cohort. The low and high patient groups were formed based on the optimal cutpoint (Supplementary table S5).

Fig. 3: AML blast cells from samples that had relapse depicted differences in transcriptome profile in comparison to samples that had CCR. A. Split UMAP plot of Dx AML blast cells of relapse- and CCR-associated samples. Blast cells formed 15 clusters based on transcriptome profile and were labeled manually based on top genes. B. Stacked bar plots showing the 
proportion of cells from each patient in different clusters (Red: Relapse-associated, Blue: CCRassociated). C. Heatmap of top differentially expressed genes between relapse-associated (clusters 0, 2, 7, 13) and CCR-associated (clusters 1, 3, 4) samples clusters. D. Kaplan Meier plots of genes in AML blasts enriched in relapse-associated samples, such as FLNA and RFLNB/FAM101B, were associated with poorer overall survival in TARGET AML dataset. Similar analysis on genes (MPO, TRH) with increased expression in the CCR-associated clusters depicted significantly better overall survival. E. Pathways that significantly $(P$ value <.01) activated ( $Z$ score $>1.5$ ) /inhibited $(Z$ score $<-1.5)$ in samples with relapse vs. samples with CCR. F. Upstream regulatory molecules significantly activated (orange) in the relapse- vs. CCRassociated samples.

Fig. 4: Immune microenvironment analysis of samples collected at the time of diagnosis. A. Split UMAP plot of bone marrow microenvironment cells based on relapse- and CCRassociated status of samples. B. Expression based dot plot of canonical cell specific markers used for the annotation of lymphoid, myeloid, and erythroid lineages. C. Stacked bar plots showing the proportion of cells from each patient in the different clusters (Red: Relapse, Blue: Remission).

Fig. 5: Relapse-associated samples at diagnosis depicted enrichment of $T$ cells with enhanced expression of exhaustion markers. A. t-distributed Stochastic Neighbor Embedding (t-SNE) plot depicting 15 sub-clusters of T-lymphocytes. The sub-clusters were annotated based on the canonical cell type markers: Naïve T cells $\left(C C R 7^{+}, L E F 1^{+}, T C F 7^{+}\right), C D 4^{+}$effector T cells

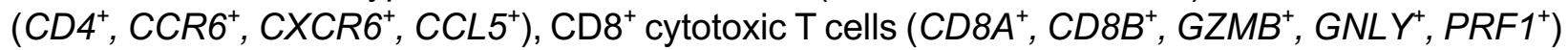
and exhausted T cells $\left(H A V C R 2^{+}, L A G 3^{+}, P D C D 1^{+}, N F A T C 1^{+}, T I G I T^{+}, T O X^{+}\right)$. B. Stacked bar plots showing cluster wise proportion of cells from each patient (Red: Relapse, Blue: CCR). C. Proportion of T cells in relapse and CCR patients. ${ }^{*}$ P-value <.05 D. Overall enrichment of Naïve and Exhausted T cell signatures across relapse and CCR samples based on ssGSEA score. E. Enrichment of Cytotoxic, IFNg- and Regulatory- T cells in the CCR and relapse predominant subclusters. ${ }^{* * * *}$ P-value <.0001 F. Heatmap of top differentially expressed genes identified by comparing the CCR and relapse dominant T cell sub-clusters $\mathbf{G}$. Pathways that are significantly activated (Z-score $>1.5$ ) and inhibited $(Z$-score $<-1.5)$ in the relapse $T$ cells associated genes. Pathways achieved $P$ value $<.01$ based on Fisher's Exact test.

Fig. 6: CCR-associated samples at diagnosis depicted enrichment of inflammatory monocytes/macrophages. A. UMAP plot depicting lassoed monocytes/macrophages clusters in Dx samples. Cluster 1 and 2 are enriched with cells from CCR patient samples whereas cluster 7 is enriched with cells from relapse samples. B. Heatmap of top DEGs from comparison of relapse- (cluster 7) and CCR- associated dominant (clusters 1, 2) clusters. C. Dot plot of key markers to characterize monocyte and macrophages subtypes. D. Upstream regulatory molecules significantly inhibited (blue) and activated (red) in the CCR-associated samples enriched monocytes/macrophages clusters in comparison to relapse-associated cluster. E. Split violin plots showing gene expression levels of specific regulators in CCR- (clusters 1,2) and relapse-associated (cluster 7) samples.

Fig. 7: scRNA-Seq analysis of EOI samples identified treatment resistant blast cells with distinct transcriptome landscape. A. UMAP plot with 13 clusters based on transcriptome profile, showing various cell types in EOI samples. B. Bar plot of 7-genes signature identifies 
clusters 6 and 9 as blast-dominant cell clusters, along with clusters 10 and 11. C. Dot plot of canonical markers used for cluster annotation. D. Heatmap of top markers that are significantly differently expressed between treatment-resistant (residual at EOI) and treatment-responsive (only at Dx cells) E. Violin plots of pathways significantly enriched in treatment-resistant blast cells compared to treatment-responsive. F. Regulators that are significantly activated (orange color) and inhibited (blue color) in treatment-resistant blast cells compared to treatmentresponsive blast cells.

Fig. 8: EOI Non-AML cells analysis exhibits different patterns in samples from those with relapse and those with CCR. (A) UMAP plot of annotated non-AML cell clusters split based on relapse and CCR status. (B) Bar plot showing the proportion of cell types in relapse and CCR samples at time of therapy induced induction. Y-axis represents fraction of each cell type. (C) Bar plot showing sample/patient representation (Y-axis) in major cell types (X-axis). (D) Bar plot of scaled SSGSEA scores and scatter plot of module scores of mono/mac cells from those with relapse and those with CCR show that M1 markers are significantly more enriched in those with CCR $(p<0.001)$ and M2 markers are more enriched in those with relapse $(p<0.05)$. (E) Bar plot of significantly different pathways in T-cell 2 cluster (relapsed-enriched) compared to T-cell 1 cluster. 


\section{References}

1. G. Egan, Y. Chopra, S. Mourad, K. Y. Chiang, J. Hitzler, Treatment of acute myeloid leukemia in children: A practical perspective. Pediatr Blood Cancer 68, e28979 (2021).

2. J. Chen, C. L. Glasser, New and Emerging Targeted Therapies for Pediatric Acute Myeloid Leukemia (AML). Children (Basel) 7, (2020).

3. H. Bolouri, J. E. Farrar, T. Triche, Jr., R. E. Ries, E. L. Lim, T. A. Alonzo, Y. Ma, R. Moore, A. J. Mungall, M. A. Marra, J. Zhang, X. Ma, Y. Liu, Y. Liu, J. M. G. Auvil, T. M. Davidsen, P. Gesuwan, L. C. Hermida, B. Salhia, S. Capone, G. Ramsingh, C. M. Zwaan, S. Noort, S. R. Piccolo, E. A. Kolb, A. S. Gamis, M. A. Smith, D. S. Gerhard, S. Meshinchi, The molecular landscape of pediatric acute myeloid leukemia reveals recurrent structural alterations and age-specific mutational interactions. Nat Med 24, 103-112 (2018).

4. E. Becht, L. Mclnnes, J. Healy, C. A. Dutertre, I. W. H. Kwok, L. G. Ng, F. Ginhoux, E. W. Newell, Dimensionality reduction for visualizing single-cell data using UMAP. Nat Biotechnol, (2018).

5. I. Khatri, M. K. Bhasin, A transcriptomics-based meta-analysis combined with machine learning identifies a secretory biomarker panel for diagnosis of pancreatic adenocarcinoma. Frontiers in genetics 11, (2020).

6. M. K. Bhasin, K. Ndebele, O. Bucur, E. U. Yee, H. H. Otu, J. Plati, A. Bullock, X. Gu, E. Castan, P. Zhang, Meta-analysis of transcriptome data identifies a novel 5-gene pancreatic adenocarcinoma classifier. Oncotarget 7, 23263 (2016).

7. M. Wang, J. Guo, L. Zhang, V. Kuek, J. Xu, J. Zou, Molecular structure, expression, and functional role of Clec11a in skeletal biology and cancers. J Cell Physiol 235, 6357-6365 (2020).

8. G. Al-Khadairi, A. Naik, R. Thomas, B. Al-Sulaiti, S. Rizly, J. Decock, PRAME promotes epithelialto-mesenchymal transition in triple negative breast cancer. J Trans/ Med 17, 9 (2019).

9. J. Dunne, C. Cullmann, M. Ritter, N. M. Soria, B. Drescher, S. Debernardi, S. Skoulakis, O. Hartmann, M. Krause, J. Krauter, A. Neubauer, B. D. Young, O. Heidenreich, siRNA-mediated AML1/MTG8 depletion affects differentiation and proliferation-associated gene expression in t(8;21)-positive cell lines and primary AML blasts. Oncogene 25, 6067-6078 (2006).

10. P. Periasamy, V. Tran, H. C. O'Neill, Identification of genes which regulate stroma-dependent in vitro hematopoiesis. PLoS One 13, e0205583 (2018).

11. M. T. Epping, L. Wang, M. J. Edel, L. Carlee, M. Hernandez, R. Bernards, The human tumor antigen PRAME is a dominant repressor of retinoic acid receptor signaling. Cell 122, 835-847 (2005).

12. Y. Xu, R. Zou, J. Wang, Z. W. Wang, X. Zhu, The role of the cancer testis antigen PRAME in tumorigenesis and immunotherapy in human cancer. Cell Prolif 53, e12770 (2020).

13. D. Szklarczyk, A. L. Gable, D. Lyon, A. Junge, S. Wyder, J. Huerta-Cepas, M. Simonovic, N. T. Doncheva, J. H. Morris, P. Bork, L. J. Jensen, C. V. Mering, STRING v11: protein-protein association networks with increased coverage, supporting functional discovery in genome-wide experimental datasets. Nucleic Acids Res 47, D607-D613 (2019).

14. D. J. Theisen, J. T. t. Davidson, C. G. Briseno, M. Gargaro, E. J. Lauron, Q. Wang, P. Desai, V. Durai, P. Bagadia, J. R. Brickner, W. L. Beatty, H. W. Virgin, W. E. Gillanders, N. Mosammaparast, M. S. Diamond, L. D. Sibley, W. Yokoyama, R. D. Schreiber, T. L. Murphy, K. M. Murphy, WDFY4 is required for cross-presentation in response to viral and tumor antigens. Science 362, 694-699 (2018).

15. C. Trapnell, D. Cacchiarelli, J. Grimsby, P. Pokharel, S. Li, M. Morse, N. J. Lennon, K. J. Livak, T. S. Mikkelsen, J. L. Rinn, The dynamics and regulators of cell fate decisions are revealed by pseudotemporal ordering of single cells. Nat Biotechnol 32, 381-386 (2014). 
16. A. Subramanian, P. Tamayo, V. K. Mootha, S. Mukherjee, B. L. Ebert, M. A. Gillette, A. Paulovich, S. L. Pomeroy, T. R. Golub, E. S. Lander, J. P. Mesirov, Gene set enrichment analysis: a knowledge-based approach for interpreting genome-wide expression profiles. Proc Natl Acad Sci U S A 102, 15545-15550 (2005).

17. V. K. Mootha, C. M. Lindgren, K. F. Eriksson, A. Subramanian, S. Sihag, J. Lehar, P. Puigserver, E. Carlsson, M. Ridderstrale, E. Laurila, N. Houstis, M. J. Daly, N. Patterson, J. P. Mesirov, T. R. Golub, P. Tamayo, B. Spiegelman, E. S. Lander, J. N. Hirschhorn, D. Altshuler, L. C. Groop, PGC1alpha-responsive genes involved in oxidative phosphorylation are coordinately downregulated in human diabetes. Nat Genet 34, 267-273 (2003).

18. Y. Mita, M. Y. Kimura, K. Hayashizaki, R. Koyama-Nasu, T. Ito, S. Motohashi, Y. Okamoto, T. Nakayama, Crucial role of CD69 in anti-tumor immunity through regulating the exhaustion of tumor-infiltrating T cells. Int Immunol 30, 559-567 (2018).

19. W. H. McBride, K. S. Iwamoto, R. Syljuasen, M. Pervan, F. Pajonk, The role of the ubiquitin/proteasome system in cellular responses to radiation. Oncogene 22, 5755-5773 (2003).

20. O. M. Seternes, A. M. Kidger, S. M. Keyse, Dual-specificity MAP kinase phosphatases in health and disease. Biochim Biophys Acta Mol Cell Res 1866, 124-143 (2019).

21. X. Xu, X. Xiong, Y. Sun, The role of ribosomal proteins in the regulation of cell proliferation, tumorigenesis, and genomic integrity. Sci China Life Sci 59, 656-672 (2016).

22. S. Chen, S. Bonifati, Z. Qin, C. St Gelais, K. M. Kodigepalli, B. S. Barrett, S. H. Kim, J. M. Antonucci, K. J. Ladner, O. Buzovetsky, K. M. Knecht, Y. Xiong, J. S. Yount, D. C. Guttridge, M. L. Santiago, L. $\mathrm{Wu}$, SAMHD1 suppresses innate immune responses to viral infections and inflammatory stimuli by inhibiting the NF-kappaB and interferon pathways. Proc Natl Acad Sci U S A 115, E3798-E3807 (2018).

23. A. Lonetti, A. Pession, R. Masetti, Targeted Therapies for Pediatric AML: Gaps and Perspective. Front Pediatr 7, 463 (2019).

24. D. Steinbach, A. Schramm, A. Eggert, M. Onda, K. Dawczynski, A. Rump, I. Pastan, S. Wittig, N. Pfaffendorf, A. Voigt, F. Zintl, B. Gruhn, Identification of a set of seven genes for the monitoring of minimal residual disease in pediatric acute myeloid leukemia. Clin Cancer Res 12, 2434-2441 (2006).

25. M. S. Ong, S. Deng, C. E. Halim, W. Cai, T. Z. Tan, R. Y. Huang, G. Sethi, S. C. Hooi, A. P. Kumar, C. T. Yap, Cytoskeletal Proteins in Cancer and Intracellular Stress: A Therapeutic Perspective. Cancers (Basel) 12, (2020).

26. H. Wurzer, C. Hoffmann, A. Al Absi, C. Thomas, Actin Cytoskeleton Straddling the Immunological Synapse between Cytotoxic Lymphocytes and Cancer Cells. Cells 8, (2019).

27. D. Bates, A. Eastman, Microtubule destabilising agents: far more than just antimitotic anticancer drugs. Br J Clin Pharmacol 83, 255-268 (2017).

28. J. Baudier, Z. A. Jenkins, S. P. Robertson, The filamin-B-refilin axis - spatiotemporal regulators of the actin-cytoskeleton in development and disease. J Cell Sci 131, (2018).

29. K. Yoshioka, A. Oda, C. Notsu, T. Ohtsuka, Y. Kawai, S. Suzuki, T. Nakamura, Y. Mabuchi, Y. Matsuzaki, R. Goitsuka, Loss of the Homeodomain Transcription Factor Prep1 Perturbs Adult Hematopoiesis in the Bone Marrow. PLoS One 10, e0136107 (2015).

30. M. Risolino, N. Mandia, F. lavarone, L. Dardaei, E. Longobardi, S. Fernandez, F. Talotta, F. Bianchi, F. Pisati, L. Spaggiari, P. N. Harter, M. Mittelbronn, D. Schulte, M. Incoronato, P. P. Di Fiore, F. Blasi, P. Verde, Transcription factor PREP1 induces EMT and metastasis by controlling the TGF-beta-SMAD3 pathway in non-small cell lung adenocarcinoma. Proc Natl Acad Sci U S A 111, E3775-3784 (2014). 
31. O. Gay, B. Gilquin, F. Nakamura, Z. A. Jenkins, R. McCartney, D. Krakow, A. Deshiere, N. Assard, J. H. Hartwig, S. P. Robertson, J. Baudier, RefilinB (FAM101B) targets filamin A to organize perinuclear actin networks and regulates nuclear shape. Proc Natl Acad Sci U S A 108, 1146411469 (2011).

32. S. S. Ithychanda, D. Hsu, H. Li, L. Yan, D. D. Liu, M. Das, E. F. Plow, J. Qin, Identification and characterization of multiple similar ligand-binding repeats in filamin: implication on filaminmediated receptor clustering and cross-talk. J Biol Chem 284, 35113-35121 (2009).

33. Z. Wei, H. Xu, Y. Zhang, X. Yi, X. Yang, Y. Chen, N. Mao, S. Li, D. Xu, S. Li, H. Zhang, D. Li, G. Zhang, B. Zhang, F. Jin, X. Gao, W. Cai, L. Zhang, R. Wang, F. Yang, Rho GDP dissociation inhibitor alpha silencing attenuates silicosis by inhibiting RhoA/Rho kinase signalling. Exp Cell Res 380, 131-140 (2019).

34. J. Penarando, L. M. Lopez-Sanchez, R. Mena, S. Guil-Luna, F. Conde, V. Hernandez, M. Toledano, V. Gudino, M. Raponi, C. Billard, C. Villar, C. Diaz, J. Gomez-Barbadillo, J. De la Haba-Rodriguez, K. Myant, E. Aranda, A. Rodriguez-Ariza, A role for endothelial nitric oxide synthase in intestinal stem cell proliferation and mesenchymal colorectal cancer. BMC Biol 16, 3 (2018).

35. X. Li, Y. Dai, B. Chen, J. Huang, S. Chen, L. Jiang, Clinical significance of CD34(+)CD117(dim)/CD34(+)CD117(bri) myeloblast-associated gene expression in $t(8 ; 21)$ acute myeloid leukemia. Front Med, (2021).

36. J. L. Quintanar, I. Guzman-Soto, Hypothalamic neurohormones and immune responses. Front Integr Neurosci 7, 56 (2013).

37. H. Itonaga, D. Imanishi, Y. F. Wong, S. Sato, K. Ando, Y. Sawayama, D. Sasaki, K. Tsuruda, H. Hasegawa, Y. Imaizumi, J. Taguchi, H. Tsushima, S. Yoshida, T. Fukushima, T. Hata, Y. Moriuchi, K. Yanagihara, Y. Miyazaki, Expression of myeloperoxidase in acute myeloid leukemia blasts mirrors the distinct DNA methylation pattern involving the downregulation of DNA methyltransferase DNMT3B. Leukemia 28, 1459-1466 (2014).

38. C. C. Lin, Y. C. Hsu, Y. H. Li, Y. Y. Kuo, H. A. Hou, K. H. Lan, T. C. Chen, Y. S. Tzeng, Y. Y. Kuo, C. J. Kao, P. H. Chuang, M. H. Tseng, Y. C. Chiu, W. C. Chou, H. F. Tien, Higher HOPX expression is associated with distinct clinical and biological features and predicts poor prognosis in de novo acute myeloid leukemia. Haematologica 102, 1044-1053 (2017).

39. S. P. Short, C. S. Williams, Selenoproteins in Tumorigenesis and Cancer Progression. Adv Cancer Res 136, 49-83 (2017).

40. A. H. Elsayed, R. Rafiee, X. Cao, S. Raimondi, J. R. Downing, R. Ribeiro, Y. Fan, T. A. Gruber, S. Baker, J. KIco, J. E. Rubnitz, S. Pounds, J. K. Lamba, A six-gene leukemic stem cell score identifies high risk pediatric acute myeloid leukemia. Leukemia 34, 735-745 (2020).

41. B. M. Stevens, C. L. Jones, D. A. Pollyea, R. Culp-Hill, A. D'Alessandro, A. Winters, A. Krug, D. Abbott, M. Goosman, S. Pei, H. Ye, A. E. Gillen, M. W. Becker, M. R. Savona, C. Smith, C. T. Jordan, Fatty acid metabolism underlies venetoclax resistance in acute myeloid leukemia stem cells. Nat Cancer 1, 1176-1187 (2020).

42. C. P. Burns, S. P. Wei, A. A. Spector, Fatty acid metabolism in L1210 murine leukemia cells: differences in modification of fatty acids incorporated into various lipids. Lipids 13, 666-672 (1978).

43. P. van Galen, V. Hovestadt, M. H. Wadsworth Ii, T. K. Hughes, G. K. Griffin, S. Battaglia, J. A. Verga, J. Stephansky, T. J. Pastika, J. Lombardi Story, G. S. Pinkus, O. Pozdnyakova, I. Galinsky, R. M. Stone, T. A. Graubert, A. K. Shalek, J. C. Aster, A. A. Lane, B. E. Bernstein, Single-Cell RNA-Seq Reveals AML Hierarchies Relevant to Disease Progression and Immunity. Cell 176, 1265-1281 e1224 (2019).

44. R. Austin, M. J. Smyth, S. W. Lane, Harnessing the immune system in acute myeloid leukaemia. Crit Rev Oncol Hematol 103, 62-77 (2016). 
45. M. Deng, X. Gui, J. Kim, L. Xie, W. Chen, Z. Li, L. He, Y. Chen, H. Chen, W. Luo, Z. Lu, J. Xie, H. Churchill, Y. Xu, Z. Zhou, G. Wu, C. Yu, S. John, K. Hirayasu, N. Nguyen, X. Liu, F. Huang, L. Li, H. Deng, H. Tang, A. H. Sadek, L. Zhang, T. Huang, Y. Zou, B. Chen, H. Zhu, H. Arase, N. Xia, Y. Jiang, R. Collins, M. J. You, J. Homsi, N. Unni, C. Lewis, G. Q. Chen, Y. X. Fu, X. C. Liao, Z. An, J. Zheng, N. Zhang, C. C. Zhang, LILRB4 signalling in leukaemia cells mediates $T$ cell suppression and tumour infiltration. Nature 562, 605-609 (2018).

46. Y. Han, Y. Dong, Q. Yang, W. Xu, S. Jiang, Z. Yu, K. Yu, S. Zhang, Acute Myeloid Leukemia Cells Express ICOS Ligand to Promote the Expansion of Regulatory T Cells. Front Immunol 9, 2227 (2018).

47. Y. S. Al-Matary, L. Botezatu, B. Opalka, J. M. Hones, R. F. Lams, A. Thivakaran, J. Schutte, R. Koster, K. Lennartz, T. Schroeder, R. Haas, U. Duhrsen, C. Khandanpour, Acute myeloid leukemia cells polarize macrophages towards a leukemia supporting state in a Growth factor independence 1 dependent manner. Haematologica 101, 1216-1227 (2016).

48. A. Butler, P. Hoffman, P. Smibert, E. Papalexi, R. Satija, Integrating single-cell transcriptomic data across different conditions, technologies, and species. Nat Biotechnol 36, 411-420 (2018).

49. I. Korsunsky, N. Millard, J. Fan, K. Slowikowski, F. Zhang, K. Wei, Y. Baglaenko, M. Brenner, P. R. Loh, S. Raychaudhuri, Fast, sensitive and accurate integration of single-cell data with Harmony. Nat Methods 16, 1289-1296 (2019).

50. L. J. P. v. d. Maaten, G. E. Hinton, Visualizing High-Dimensional Data Using t-SNE. Journal of Machine Learning Research, 2579-2605 (2008).

51. M. E. Ritchie, B. Phipson, D. Wu, Y. Hu, C. W. Law, W. Shi, G. K. Smyth, limma powers differential expression analyses for RNA-sequencing and microarray studies. Nucleic Acids Res 43, e47 (2015).

52. C. W. Law, Y. Chen, W. Shi, G. K. Smyth, voom: Precision weights unlock linear model analysis tools for RNA-seq read counts. Genome Biol 15, R29 (2014).

53. C. W. Law, M. Alhamdoosh, S. Su, X. Dong, L. Tian, G. K. Smyth, M. E. Ritchie, RNA-seq analysis is easy as 1-2-3 with limma, Glimma and edgeR. F1000Res 5, (2016).

54. C. Contal, J. O'Quigley, An application of changepoint methods in studying the effect of age on survival in breast cancer. Computational Statistics \&amp; Data Analysis 30, 253-270 (1999).

55. S. Hanzelmann, R. Castelo, J. Guinney, GSVA: gene set variation analysis for microarray and RNAseq data. BMC Bioinformatics 14, 7 (2013).

56. X. Gao, D. Hu, M. Gogol, H. Li, ClusterMap: compare multiple single cell RNA-Seq datasets across different experimental conditions. Bioinformatics 35, 3038-3045 (2019).

Author contributions: HSS, SIP, DD, SR, MP, RJS, SMC, DSW, CCP, DKG collected bone marrow samples and clinical data. BET, SSB and MB designed the experiments. BET, SSB conducted the experiments. GBU, PP, BET, DS, HM, SSB, BD, KG, MB analyzed data. GBU, PP, $B E T, D S, S S B, B D, R J S, S I P, C C P$, DKG interpreted results and wrote manuscript. MB supervised the study.

\section{Acknowledgments:}

The study is supported through funding from Cure Childhood Cancer Foundation (DKG) and Emory startup funds (MKB). 
Conflict of interest statement: An invention disclosure for 7-gene AML blast signature has been submitted (MKB). MKB has equity in Canomiks Inc and on its board as chief scientific advisor. The authors (DKG, DD) hold equity in Meryx incorporated.

\section{Data availability:}

ScRNASeq data will be submitted to NCBI's Gene Expression Omnibus (GEO). An interactive data resource and analytical tool developed based on this AML single cell data is available online at https://bhasinlab.bmi.emory.edu/PediatricSC/. 
A.

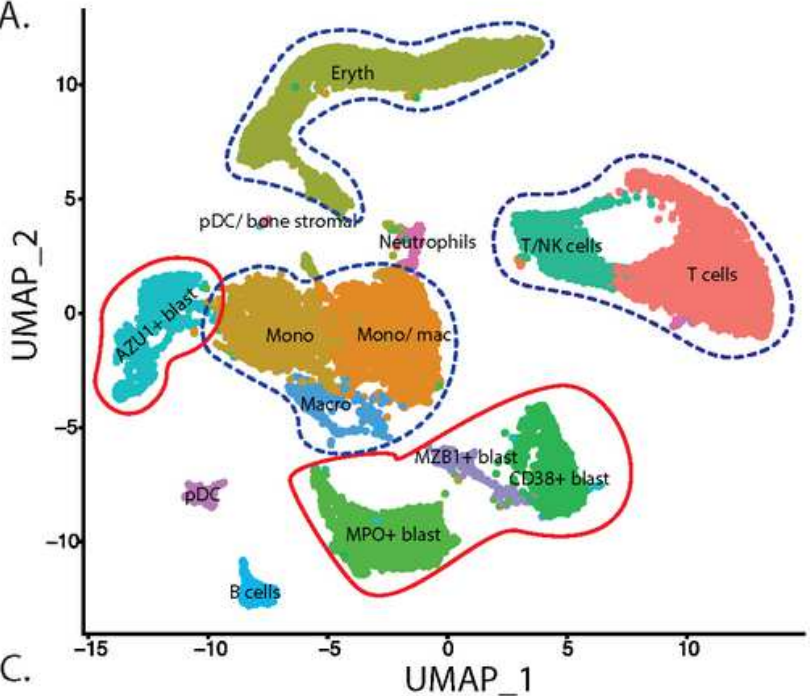

C.

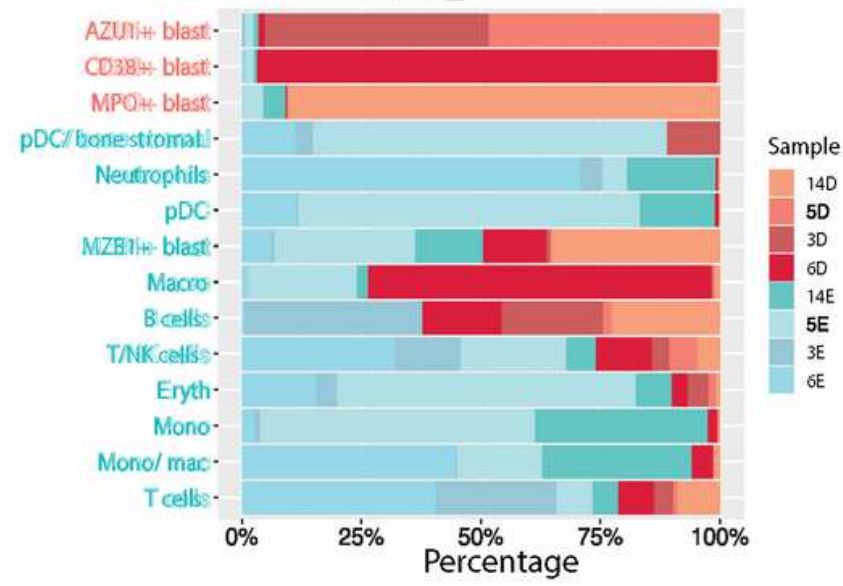

E.

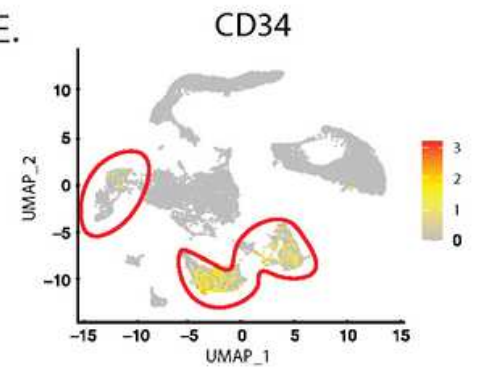

B.

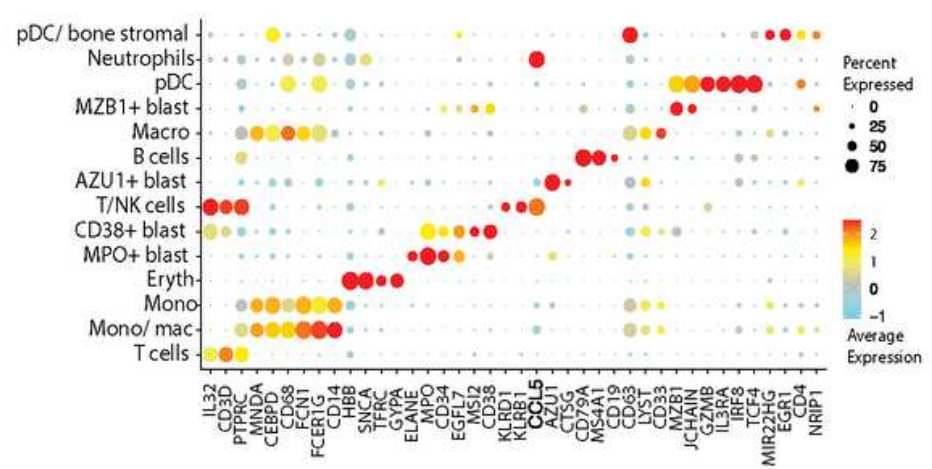

Diagnosis (Dx)

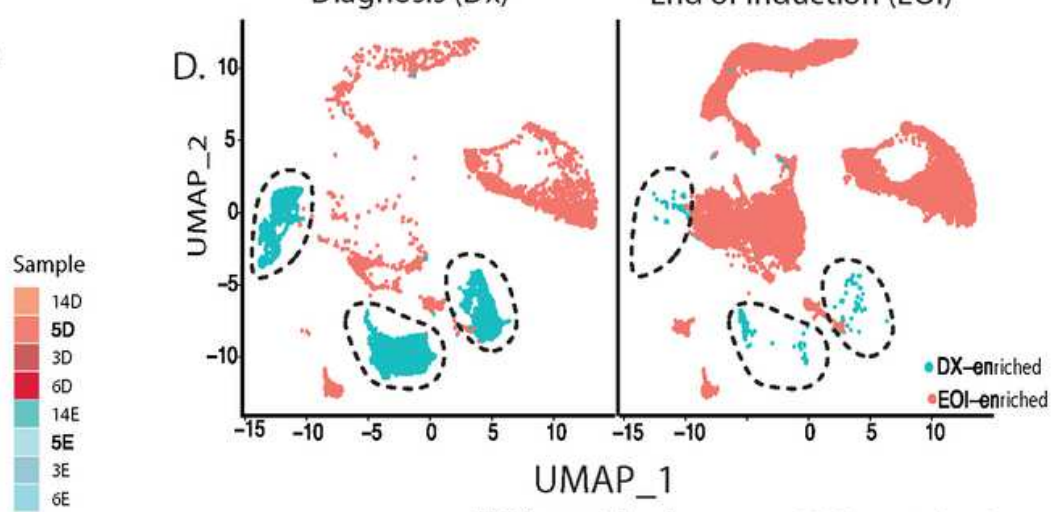

Figure 1

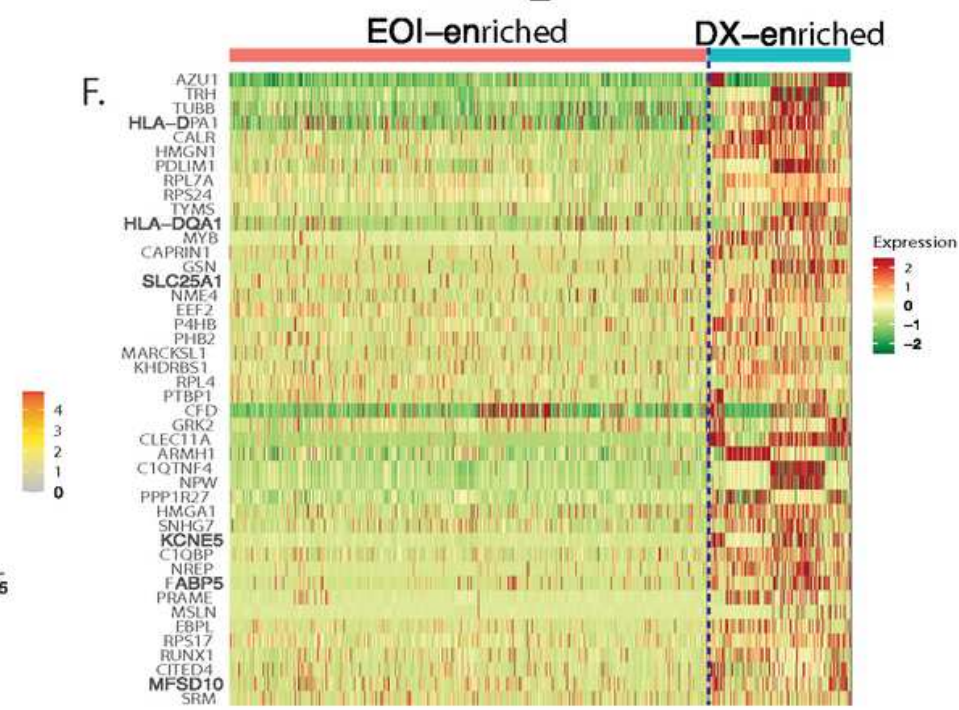

Figure 1

Single cell transcriptional profiling identifies three heterogenous putative AML blast cell clusters by comparative profiling of paired Diagnostics (Dx) and End of Induction (EOI) bone marrow samples. A. UMAP embedding of the paired Dx and EOI samples consisting of 19,350 high quality single cells portioned into 14 clusters. These clusters are colored based on canonical cell types gene markers (Fig. 1B) that include T cell (CD3D+, IL32+), B cells (CD19+, CD79A+, MS4A1+), monocyte (CD14+), macrophages (CD68+), macrophage/monocyte (CD14+, CD68+), plasmacytoid dendritic cells-pDC 
(GZMB+, IL3RA+, IRF8+), and neutrophils (CD63+). The blast cells were annotated based on expression of top overexpressed gene. B. Dot plot showing expression of different cell type specific marker genes that were used to annotate the cell types (Red: high, Yellow: Mid, Cyan: low). C. Relative proportion of cells of different samples in each cell type cluster. Most of the blast cell clusters are made up of cells from 1-2 patient samples indicating AML blast heterogeneity. On the other hand, most of the immune cell clusters contain cells from multiple patient samples. D. Split UMAP showing the putative undifferentiated blast cells that are significantly over-represented in Dx samples (Dx enriched) and reduced in the EOI samples. E. Expression of clinically validated blast cell markers (MPO and CD34) across single cell clusters. Clusters with positive expression of marker genes are lassoed. CD34 expression is highly heterogenous. $\mathrm{F}$. Heatmap of selected set of 44 genes showing significant overexpression (Fold Change $>1.2$, Adjusted $P$ value <.01) in Dx Enriched blast cells in comparison of other differentiated cell types. Relative gene expression is shown in pseudo color, where green represents downregulation, and red represents upregulation. 
Figure 2

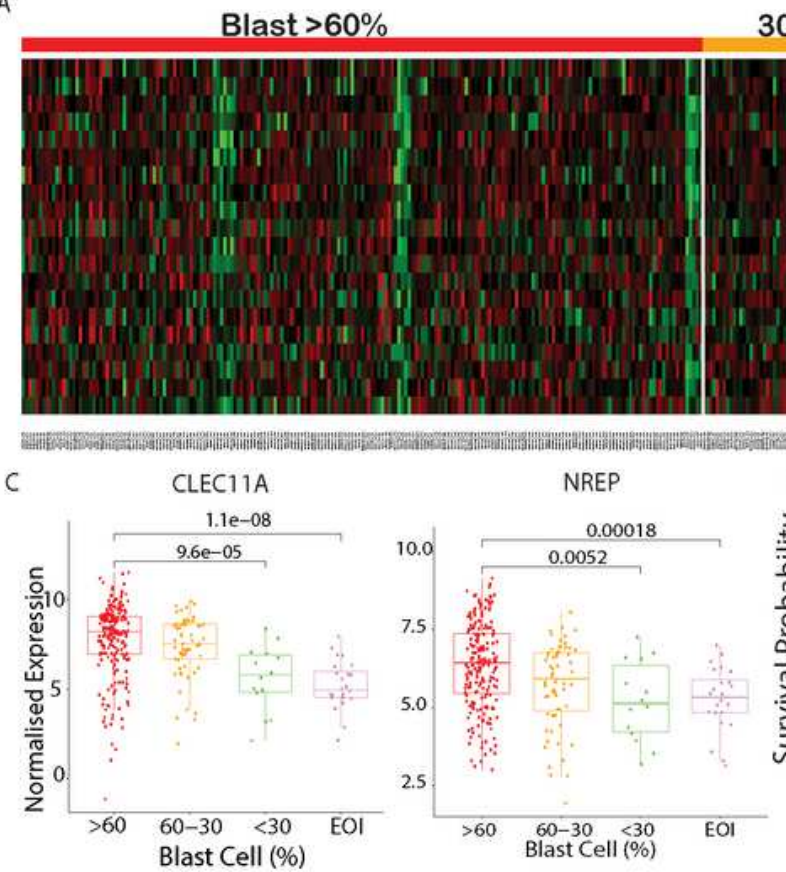

E.

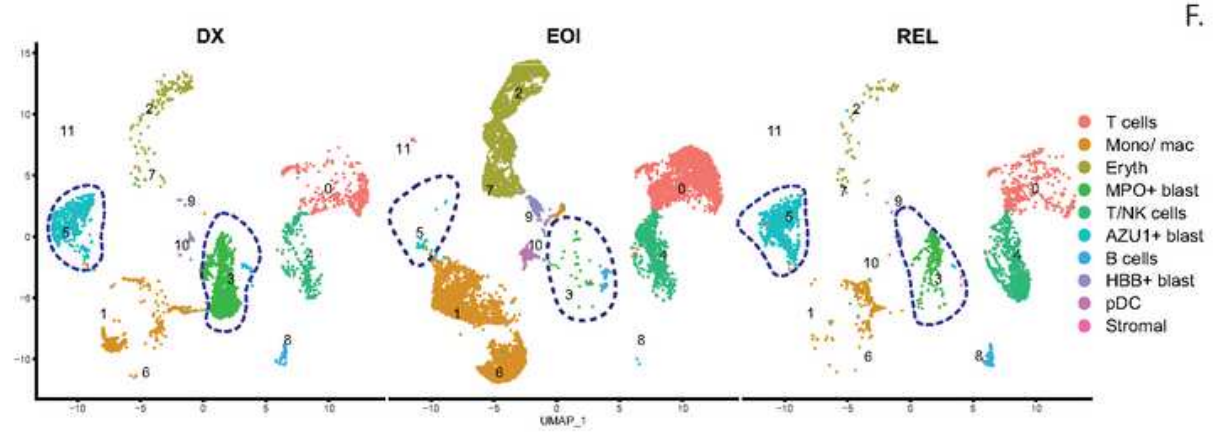

G.

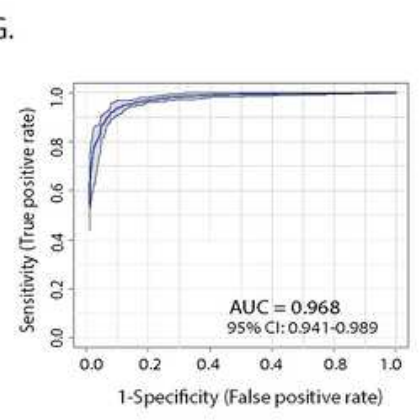

$30-60 \%<30 \%$ EOI
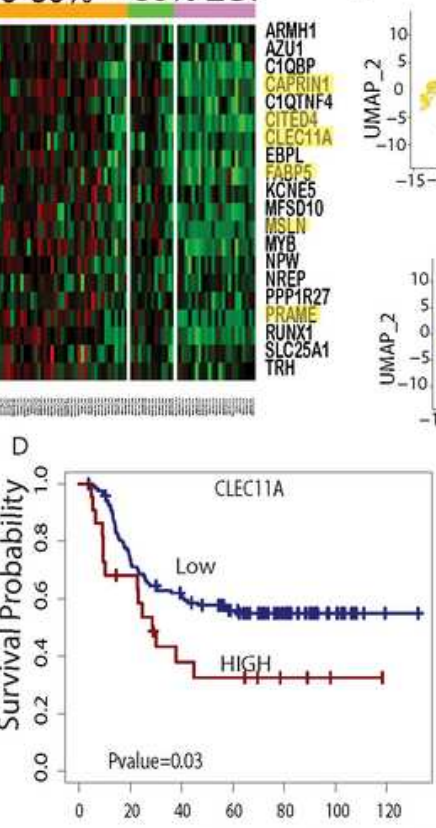

Overall Survival (Months)

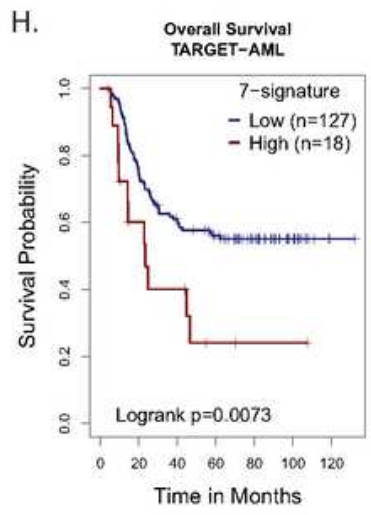

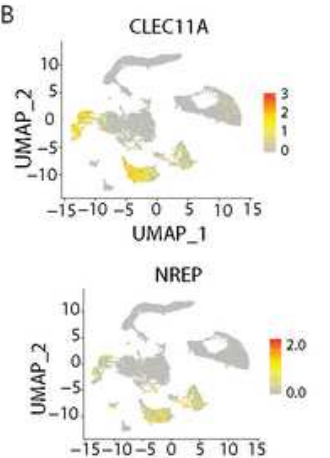

UMAP_1

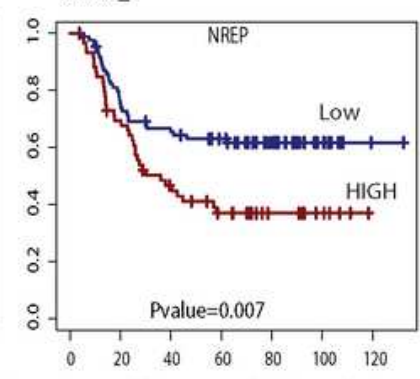

Overall Survival (Months)

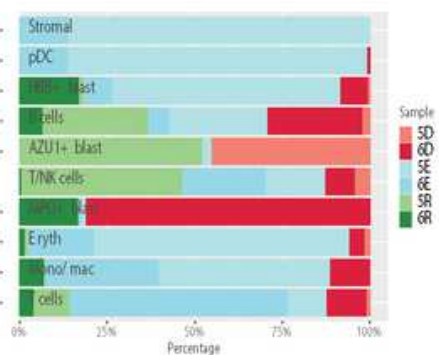

Figure 2

Development of blast progenitor signature from heterogenous AML blast cells. A. Heatmap of 20 genes that depicts significant overexpression in the TARGET AML samples with high blast enrichment (>60\%) as compared to low blast (<30\%) and EOI samples. B. Feature plot of selected genes (CLEC11A, NREP) from 20 gene signature showing uniform overexpression in the heterogenous blast clusters. C.

Representative expression profiles and D. survival correlations of select genes (CLEC11A, NREP) showing 
a progressive downregulation pattern from high blast enrichment to low blast and EOI TARGET AML samples. The significance of the enrichment was calculated using Wilcox Rank t-test ( ${ }^{*} p$-value $<0.05$, ** $\mathrm{p}$-value $<0.01$, ${ }^{\star * *} \mathrm{p}$-value $\left.<0.001\right)$. The higher expression of these CLEC11A $(\mathrm{HR}=1.9,95 \% \mathrm{Cl}: 1.1-3.4$, $\mathrm{P}=0.03)$ and NREP ( $\mathrm{HR}=1.9,95 \% \mathrm{Cl}: 1.2-3.1, \mathrm{P}=0.007)$ is associated with poor survival. E. UMAP plot depicting the blast cells identification based on the newly identified 20-gene overexpressed in blast cells (lassoed) of the longitudinal samples (DX, EOI, REL) from two patients. F. Bar plot showing fraction of cell types in DX, EOI and Rel samples. G. Receiver operative Curve (ROC) depicting performance of Support Vector Machine (SVM) approach based 7 genes signature (CLEC11A, PRAME, AZU1, NREP, ARMH1, C1QBP, TRH) to discriminated AML blast cells from other cells. H. Survival analysis depicted that patient with higher gene set enrichment score based on identified 7 genes exhibited poorer overall survival $(\mathrm{HR}=2.3,95 \% \mathrm{Cl}=1.2-4.3, \mathrm{P}=0.0073)$ in the TARGET AML cohort. The low and high patient groups were formed based on the optimal cutpoint (Supplementary table S5). 


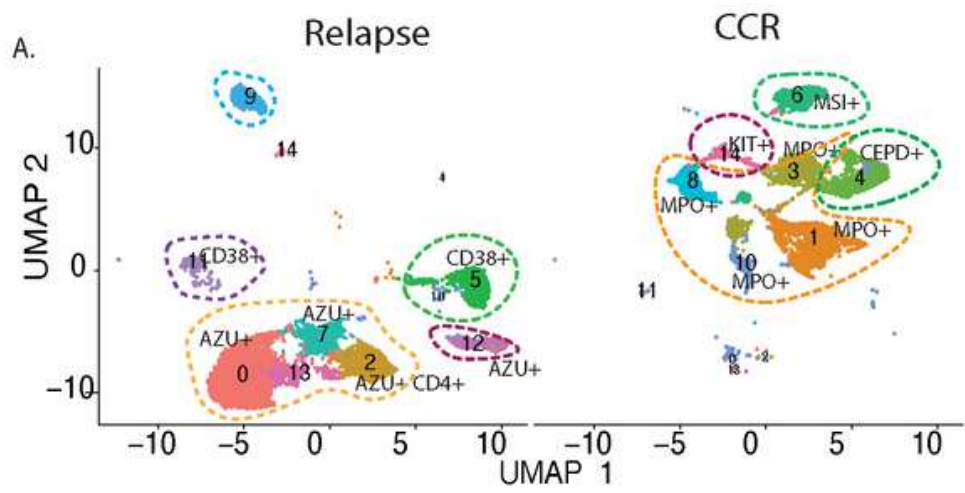

B.

Figure 3

c.
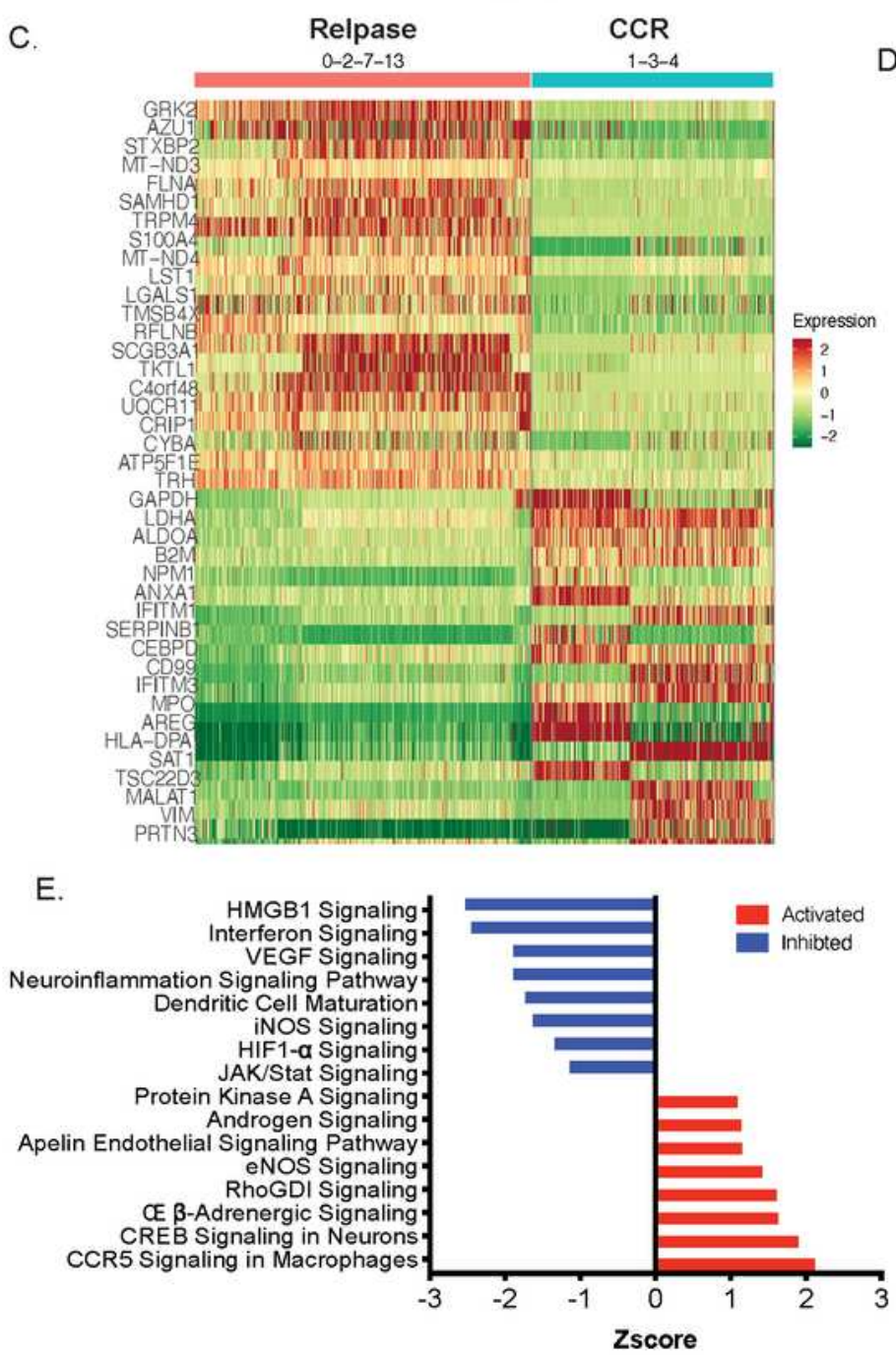

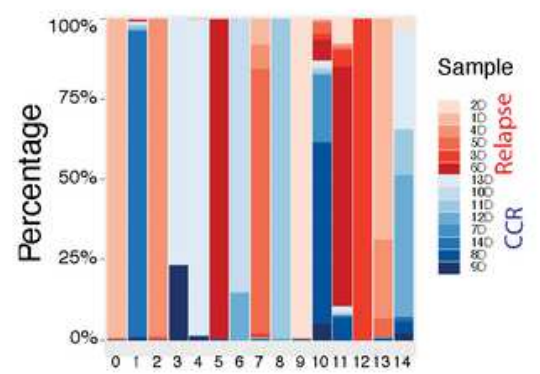

D.
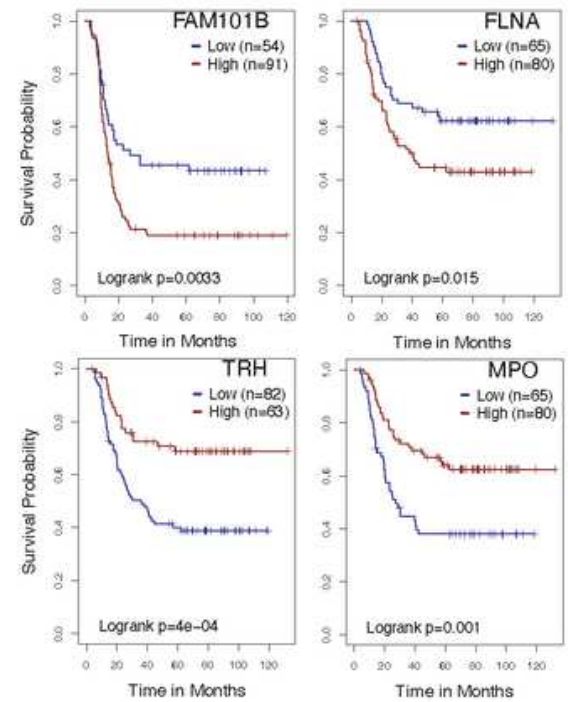

Time in Months

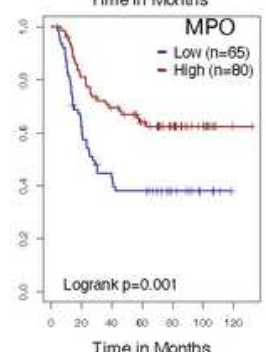

F.

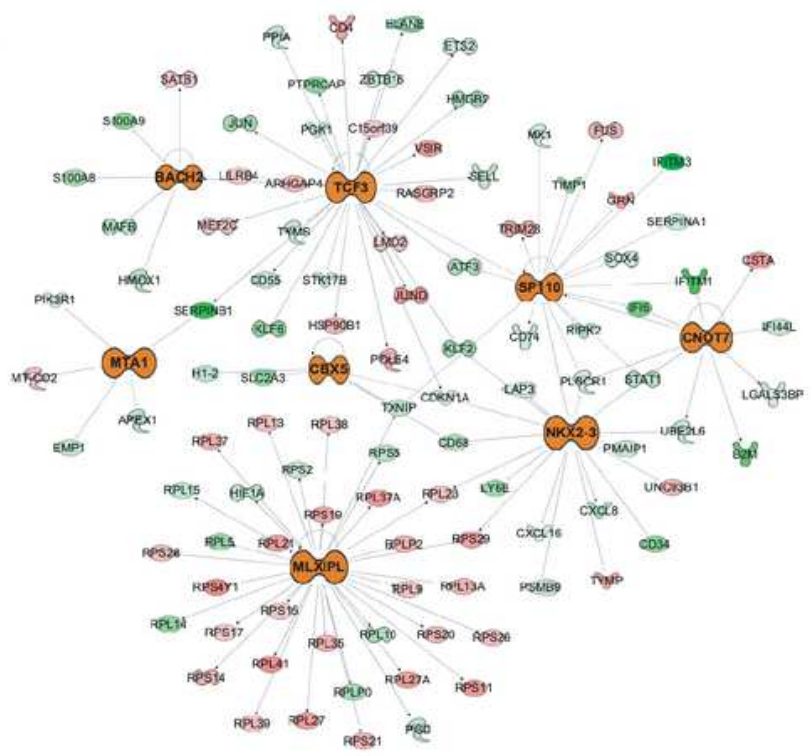

\section{Figure 3}

AML blast cells from samples that had relapse depicted differences in transcriptome profile in comparison to samples that had CCR. A. Split UMAP plot of Dx AML blast cells of relapse- and CCRassociated samples. Blast cells formed 15 clusters based on transcriptome profile and were labeled manually based on top genes. B. Stacked bar plots showing the proportion of cells from each patient in different clusters (Red: Relapse-associated, Blue: CCRassociated). C. Heatmap of top differentially 
expressed genes between relapse-associated (clusters $0,2,7,13$ ) and CCR-associated (clusters 1, 3, 4) samples clusters. D. Kaplan Meier plots of genes in AML blasts enriched in relapse-associated samples, such as FLNA and RFLNB/FAM101B, were associated with poorer overall survival in TARGET AML dataset. Similar analysis on genes (MPO, TRH) with increased expression in the CCR-associated clusters depicted significantly better overall survival. E. Pathways that significantly $(P$ value $<.01)$ activated $(Z$ score $>1.5$ ) /inhibited (Z score <-1.5) in samples with relapse vs. samples with CCR. F. Upstream regulatory molecules significantly activated (orange) in the relapse- vs. CCRassociated samples.

A.

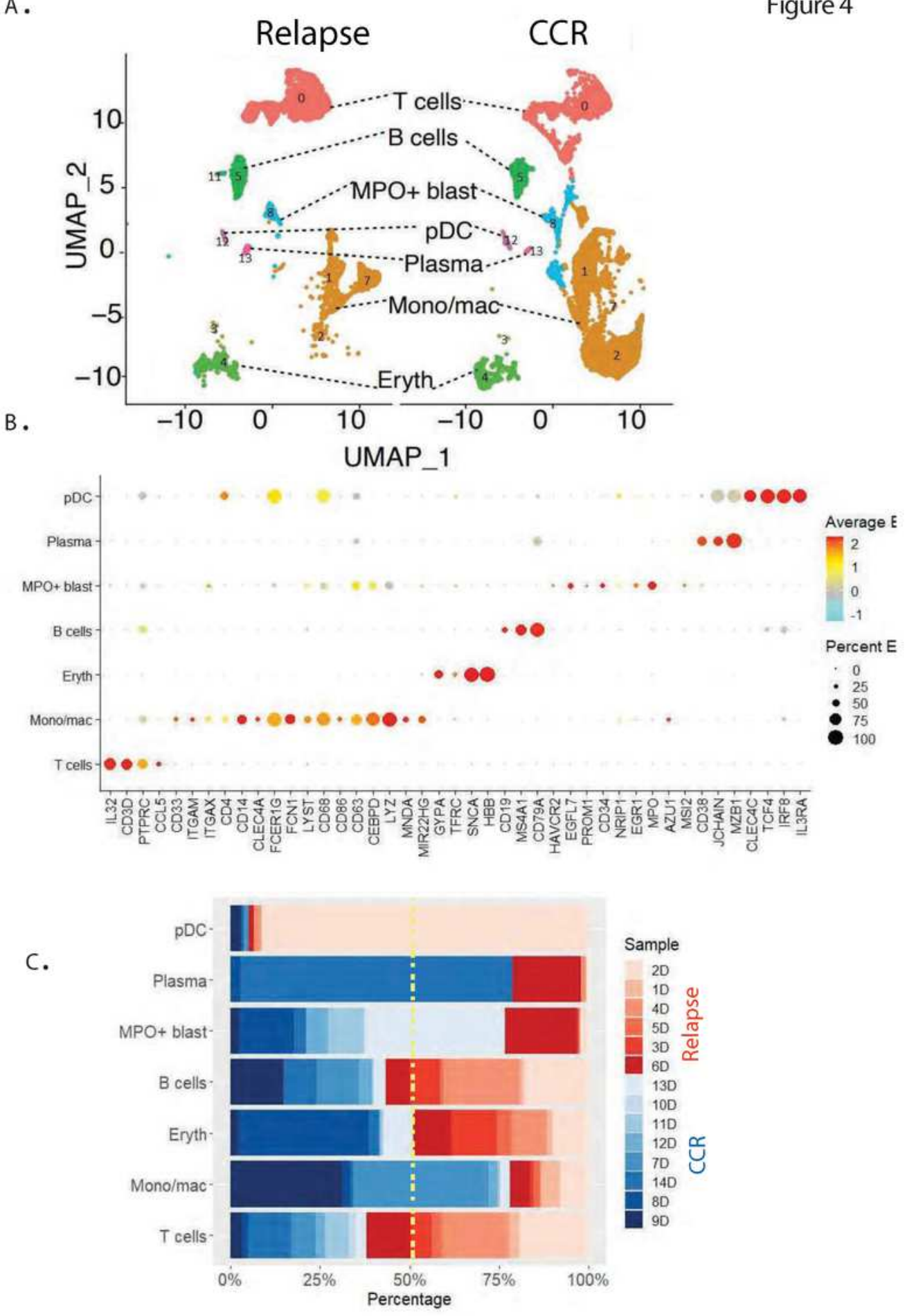


Figure 4

Immune microenvironment analysis of samples collected at the time of diagnosis. A. Split UMAP plot of bone marrow microenvironment cells based on relapse- and CCRassociated status of samples. B. Expression based dot plot of canonical cell specific markers used for the annotation of lymphoid, myeloid, and erythroid lineages. C. Stacked bar plots showing the proportion of cells from each patient in the different clusters (Red: Relapse, Blue: Remission).

A.

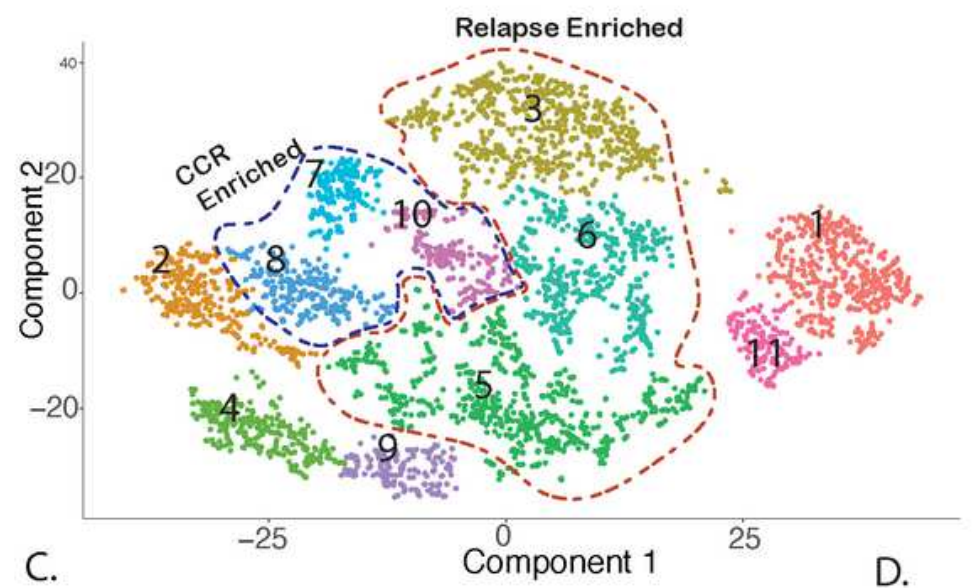

B.

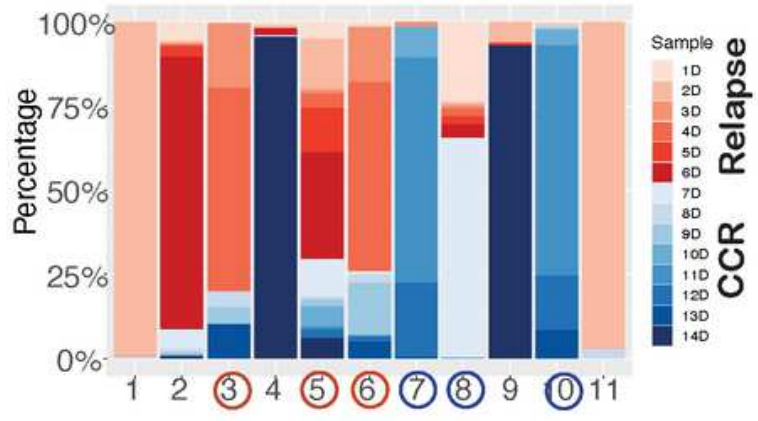

Figure 5 8
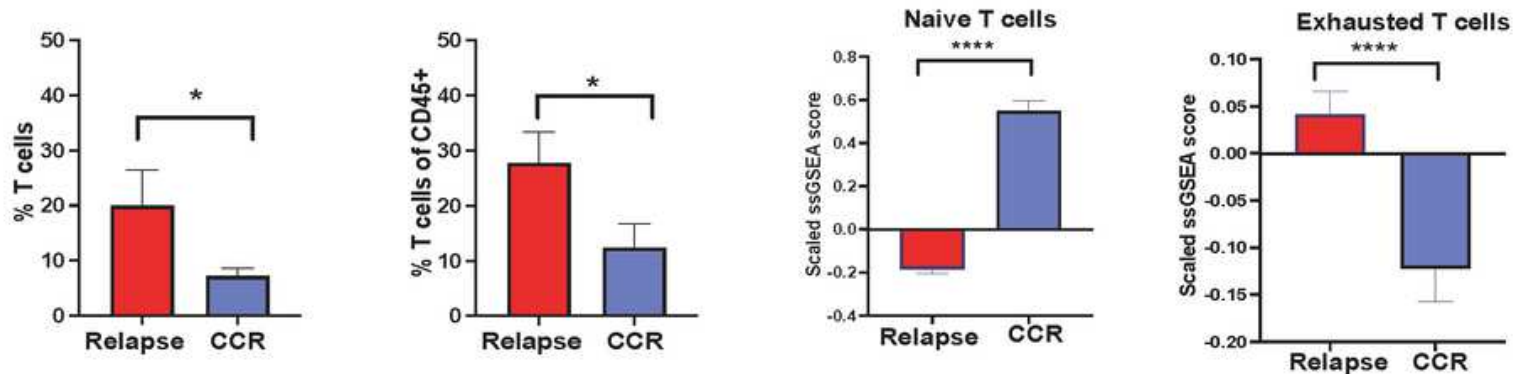

E.

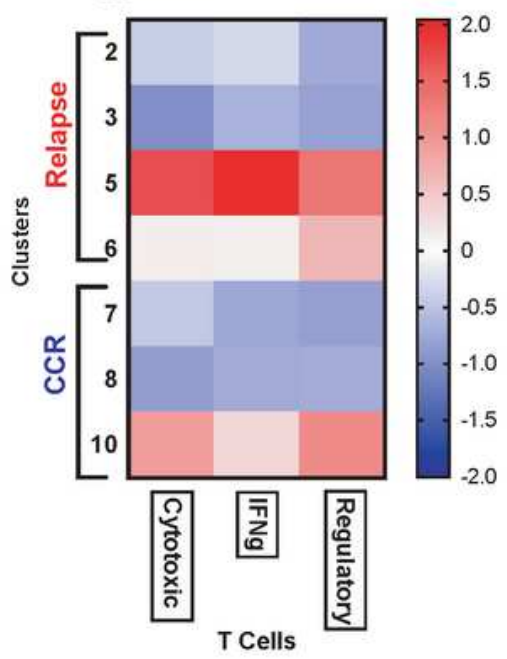

F.

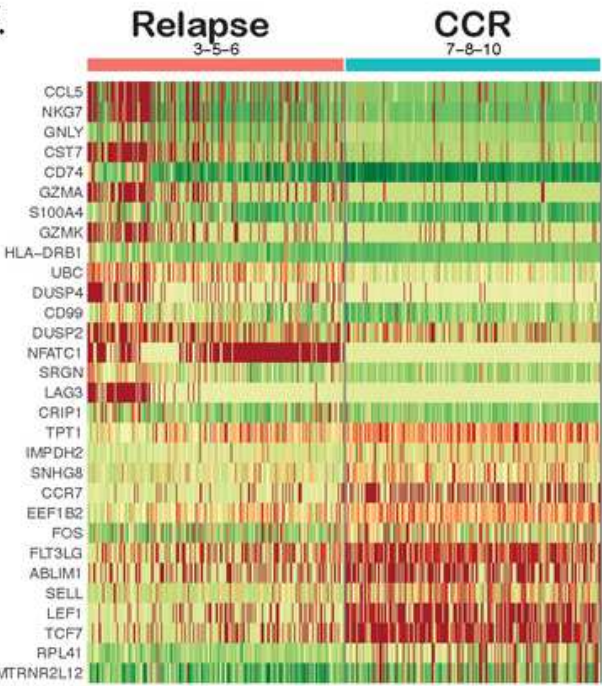

G.

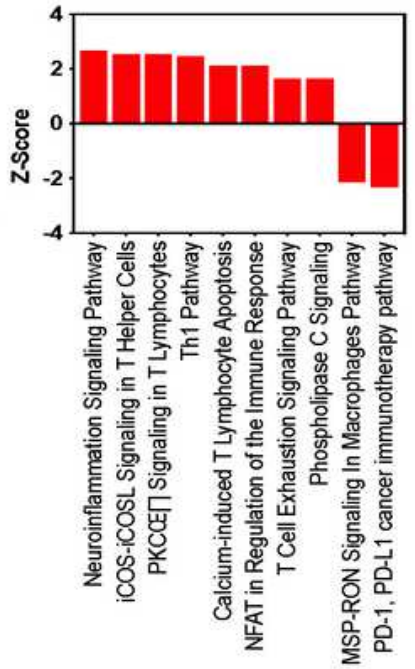




\section{Figure 5}

Relapse-associated samples at diagnosis depicted enrichment of T cells with enhanced expression of exhaustion markers. A. t-distributed Stochastic Neighbor Embedding (t-SNE) plot depicting 15 subclusters of T-lymphocytes. The sub-clusters were annotated based on the canonical cell type markers: Naïve T cells (CCR7+, LEF1+, TCF7+), CD4+ effector T cells (CD4+, CCR6+, CXCR6+, CCL5+), CD8+ cytotoxic T cells (CD8A+, CD8B+, GZMB+, GNLY+, PRF1+) and exhausted T cells (HAVCR2+, LAG3+, PDCD1+, NFATC1+, TIGIT+, TOX+). B. Stacked bar plots showing cluster wise proportion of cells from each patient (Red: Relapse, Blue: CCR). C. Proportion of T cells in relapse and CCR patients. * P-value $<.05$ D. Overall enrichment of Naïve and Exhausted T cell signatures across relapse and CCR samples based on sSGSEA score. E. Enrichment of Cytotoxic, IFNg- and Regulatory- T cells in the CCR and relapse predominant subclusters. ${ }^{\star \star \star \star}$ P-value $<.0001 \mathrm{~F}$. Heatmap of top differentially expressed genes identified by comparing the CCR and relapse dominant $T$ cell sub-clusters $\mathrm{G}$. Pathways that are significantly activated (Z-score $>1.5$ ) and inhibited (Z-score $<-1.5$ ) in the relapse T cells associated genes. Pathways achieved $P$ value $<.01$ based on Fisher's Exact test. 
A.

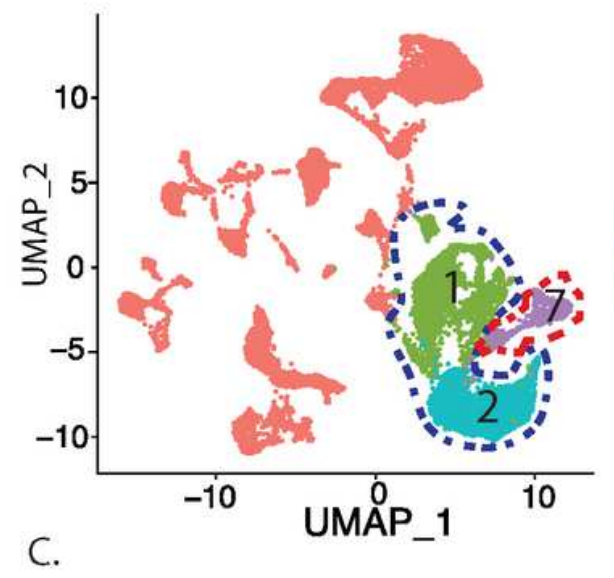

- Other

- CCR

-Relapse

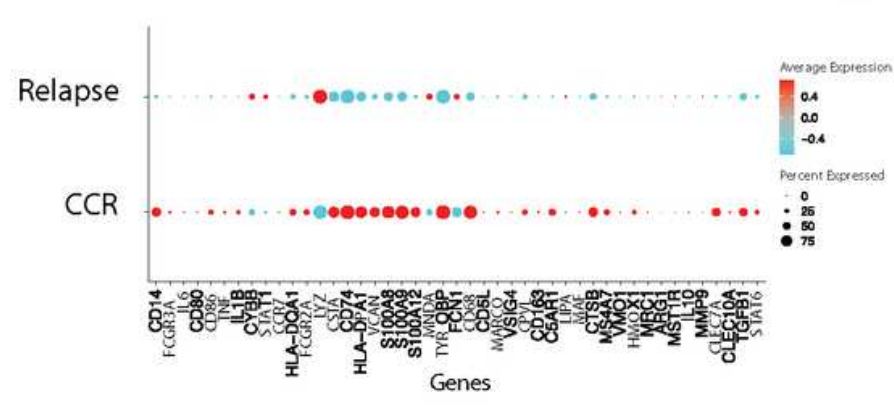

E.

D. b.

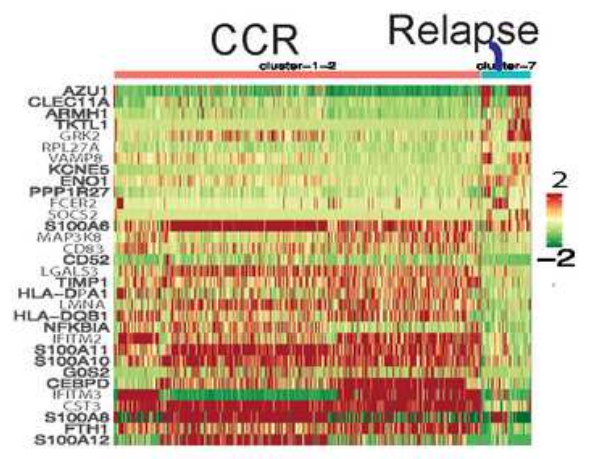

Figure 6

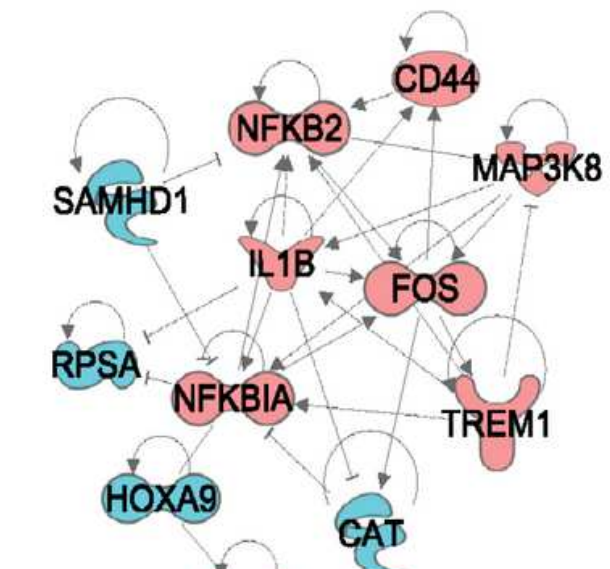

SATB1

CAT

HOXA9

SAMHD1

IL1B

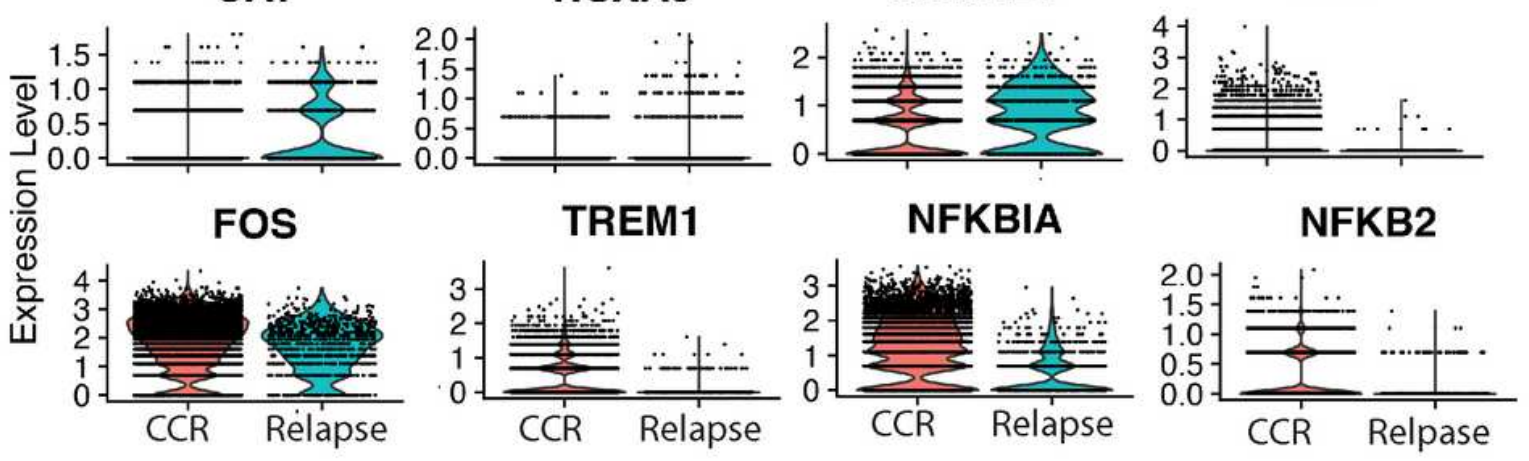

Figure 6

CCR-associated samples at diagnosis depicted enrichment of inflammatory monocytes/macrophages. A. UMAP plot depicting lassoed monocytes/macrophages clusters in Dx samples. Cluster 1 and 2 are enriched with cells from CCR patient samples whereas cluster 7 is enriched with cells from relapse samples. B. Heatmap of top DEGs from comparison of relapse- (cluster 7) and CCR- associated dominant (clusters 1,2) clusters. C. Dot plot of key markers to characterize monocyte and macrophages subtypes. D. Upstream regulatory molecules significantly inhibited (blue) and activated (red) in the CCR-associated samples enriched monocytes/macrophages clusters in comparison to relapse-associated cluster. E. Split violin plots showing gene expression levels of specific regulators in CCR- (clusters 1,2$)$ and relapseassociated (cluster 7) samples. 
A

A

C
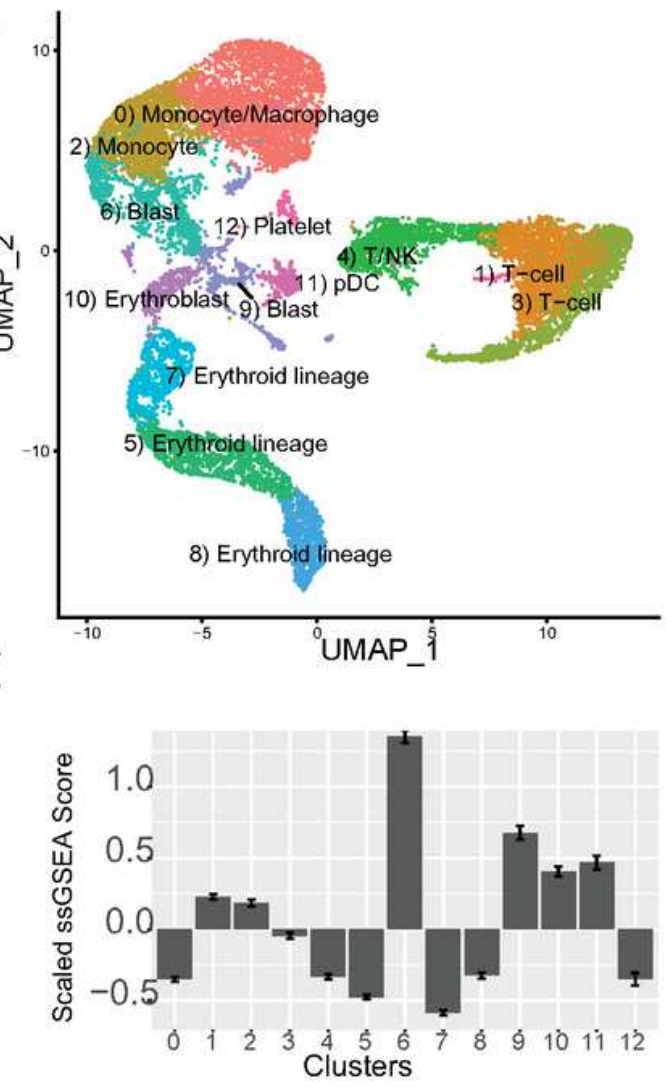

$E$
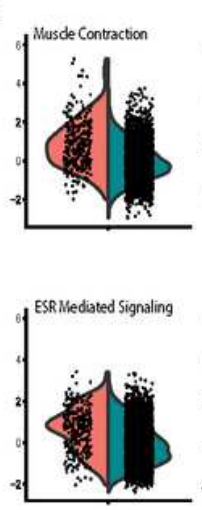

B

Figure 7
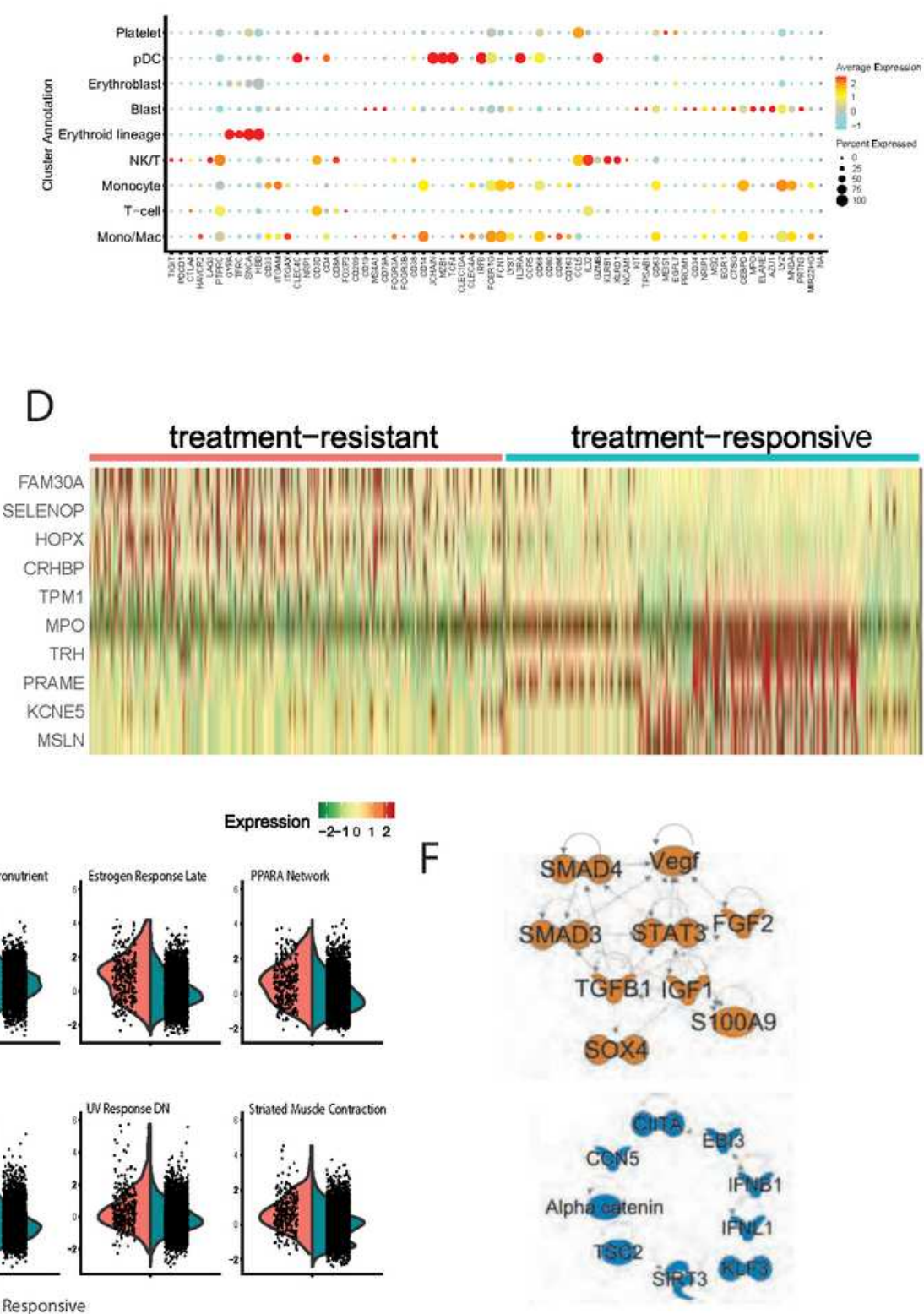

Figure 7

scRNA-Seq analysis of EOI samples identified treatment resistant blast cells with distinct transcriptome landscape. A. UMAP plot with 13 clusters based on transcriptome profile, showing various cell types in EOI samples. B. Bar plot of 7-genes signature identifies clusters 6 and 9 as blast-dominant cell clusters, along with clusters 10 and 11. C. Dot plot of canonical markers used for cluster annotation. D. Heatmap of top markers that are significantly differently expressed between treatment-resistant (residual at EOI) and treatment-responsive (only at Dx cells) E. Violin plots of pathways significantly enriched in treatmentresistant blast cells compared to treatment-responsive. F. Regulators that are significantly activated 
(orange color) and inhibited (blue color) in treatment-resistant blast cells compared to treatmentresponsive blast cells.

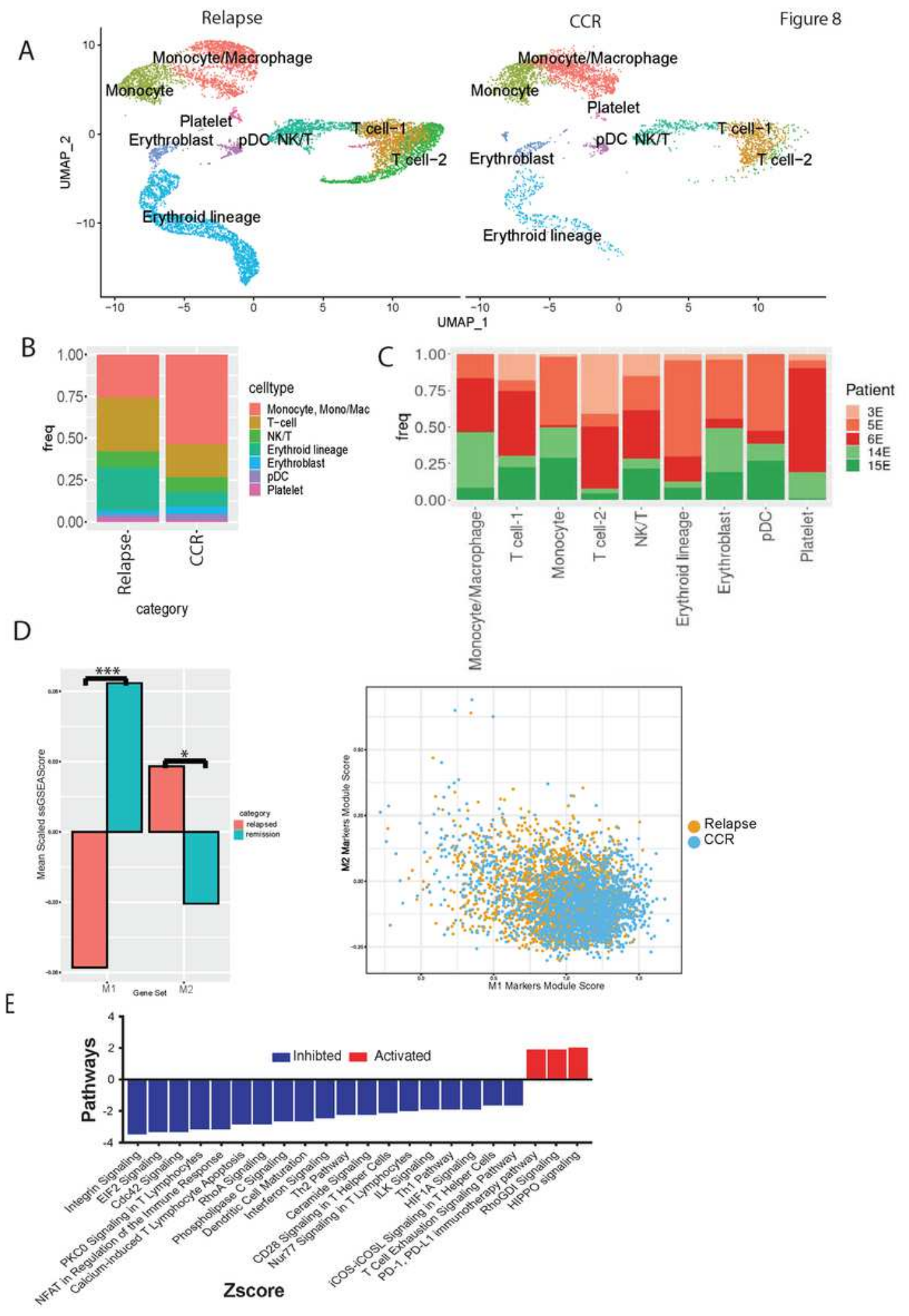

Figure 8

EOI Non-AML cells analysis exhibits different patterns in samples from those with relapse and those with CCR. (A) UMAP plot of annotated non-AML cell clusters split based on relapse and CCR status. (B) Bar plot showing the proportion of cell types in relapse and CCR samples at time of therapy induced 
induction. Y-axis represents fraction of each cell type. (C) Bar plot showing sample/patient representation (Y-axis) in major cell types (X-axis). (D) Bar plot of scaled ssGSEA scores and scatter plot of module scores of mono/mac cells from those with relapse and those with CCR show that M1 markers are significantly more enriched in those with CCR $(p<0.001)$ and M2 markers are more enriched in those with relapse $(p<0.05)$. (E) Bar plot of significantly different pathways in T-cell 2 cluster (relapsed-enriched) compared to T-cell 1 cluster.

\section{Supplementary Files}

This is a list of supplementary files associated with this preprint. Click to download.

- SupplementaryMaterialnewReferences.pdf 